\title{
Subdegree growth rates of infinite primitive permutation groups
}

\author{
Simon M. Smith \\ Dedicated to the memory of Mr J. Dodd and Mr \& Mrs L. Smith
}

\begin{abstract}
A transitive group $G$ of permutations of a set $\Omega$ is primitive if the only $G$-invariant equivalence relations on $\Omega$ are the trivial and universal relations.

If $\alpha \in \Omega$, then the orbits of the stabiliser $G_{\alpha}$ on $\Omega$ are called the $\alpha$-suborbits of $G$; when $G$ acts transitively the cardinalities of these $\alpha$-suborbits are the subdegrees of $G$.

If $G$ acts primitively on an infinite set $\Omega$, and all the suborbits of $G$ are finite, Adeleke and Neumann asked if, after enumerating the subdegrees of $G$ as a non-decreasing sequence $1=m_{0} \leq m_{1} \leq \cdots$, the subdegree growth rates of infinite primitive groups that act distancetransitively on locally finite distance-transitive graphs are extremal, and conjecture there might exist a number $c$ which perhaps depends upon $G$, perhaps only on the size of some suborbit $m$, such that $m_{r} \leq c(m-2)^{r-1}$.

In this paper it is shown that such an enumeration is not desirable, as there exist infinite primitive permutation groups possessing no infinite subdegree, in which two distinct subdegrees are each equal to the cardinality of infinitely many $\alpha$-suborbits. The examples used to show this provide several novel methods for constructing infinite primitive graphs.

A revised enumeration method is then proposed, and it is shown that, under this, Adeleke and Neumann's question may be answered, at least for groups exhibiting suitable rates of growth.
\end{abstract}

\section{Introduction}

Let $G$ be a group of permutations of an infinite set $\Omega$. If $\alpha \in \Omega$ and $g \in G$, we denote the image of $\alpha$ under $g$ by $\alpha^{g}$, and the set of images of $\alpha$ under all elements of $G$ by $\alpha^{G}$. All permutations will act on the right. We denote the stabiliser of $\alpha$ in $G$ by $G_{\alpha}$, and if $\Sigma \subseteq \Omega$ we denote the setwise and pointwise stabilisers of $\Sigma$ in $G$ by $G_{\{\Sigma\}}$ and $G_{(\Sigma)}$ respectively. A transitive group $G$ is primitive on $\Omega$ if the only $G$-invariant equivalence relations on $\Omega$ are the trivial and universal relations. It is well known that all point stabilisers in a primitive group are maximal.

Given $\alpha, \beta \in \Omega$, the set $(\alpha, \beta)^{G}$ is called an orbital of $G$. It is diagonal if $\alpha$ and $\beta$ are equal. An $\alpha$-suborbit is an orbit of $G_{\alpha}$ on $\Omega$. If $G$ is transitive on $\Omega$, the subdegrees of $G$ are the cardinalities of the $\alpha$-suborbits of $G$ for some fixed $\alpha \in \Omega$.

There is a natural pairing between orbitals of $G$ : if $\Delta:=(\alpha, \beta)^{G}$ is an orbital, then its pair $\Delta^{*}$ is the orbital $(\beta, \alpha)^{G}$. There is also a natural correspondence between the orbital $\Delta$ and the $\alpha$-suborbit $\Delta(\alpha)$, where $\Delta(\alpha):=\{\gamma \mid(\alpha, \gamma) \in \Delta\}$. For every $\alpha$-suborbit $\Upsilon$ of $G$, there is an orbital $\Delta$ such that $\Upsilon=\Delta(\alpha)$, namely the orbital $\Delta:=(\alpha, \beta)^{G}$, where $\beta$ is some vertex in $\Upsilon$. This correspondence is bijective. The pair of the $\alpha$-suborbit $\Delta(\alpha)$ is the suborbit $\Delta^{*}(\alpha)$; a suborbit or orbital is self-paired if it is equal to its pair.

2000 Mathematics Subject Classification 20B15 (primary), 05C25 (secondary). Please refer to http://www.ams.org/msc/ for a list of codes.

Publication date: 25th August, 2010. Journal reference: J. London Math. Soc. (2) 82 (2010) 526-548. 
A digraph is a directed graph without multiple edges or loops; it is a pair $(V \Gamma, A \Gamma)$, where $V \Gamma$ is the set of vertices and $A \Gamma$ the set of $\operatorname{arcs}$ of $\Gamma$. The set $A \Gamma$ consists of ordered pairs of distinct elements of $V \Gamma$. Two vertices $\alpha, \beta \in V \Gamma$ are adjacent if either $(\alpha, \beta)$ or $(\beta, \alpha)$ lies in $A \Gamma$. Throughout this paper all paths will be undirected. The distance between two connected vertices $\alpha$ and $\beta$ in $\Gamma$ is denoted by $d_{\Gamma}(\alpha, \beta)$. A digraph is locally finite if every vertex is adjacent to at most finitely many other vertices.

If $G \leq \operatorname{Sym}(\Omega)$ and $\Delta$ is an orbital of $G$, the digraph $(\Omega, \Delta)$ is called an orbital digraph of $G$. The connected components of an orbital digraph of $G$ are $G$-invariant equivalence classes on $\Omega$; thus, if $G$ is primitive, then every non-diagonal orbital digraph is connected. Diagonal digraphs have no arcs; henceforth all orbital digraphs will be non-diagonal unless otherwise stated.

Interest in the subdegree growth of infinite primitive permutation groups possessing a finite suborbit whose pair is also finite stems from the following well-known observation (see for example [1, Remark 29.8]). In [1] the result is stated only for groups possessing a finite selfpaired suborbit, but the argument holds for groups possessing a finite suborbit whose pair is also finite.

THEOREM 1.1. If $G$ is a group acting primitively on the infinite set $\Omega$, possessing a finite suborbit whose pair is also finite, then every suborbit of $G$ is finite and $\Omega$ is countable.

Henceforth, any group whose suborbits are all finite will be described as being subdegree finite. Thus a primitive group is subdegree finite if and only if it has a locally finite orbital digraph; of course, if it has a locally finite orbital digraph then all its orbital digraphs will be locally finite.

Note this theorem is not true if we merely require a primitive group $G$ to possess a finite suborbit. Indeed, in [5] Evans constructs an infinite primitive group acting on an uncountably infinite set $\Omega$, possessing a finite suborbit whose pair is infinite.

The complete digraph on $t$ vertices, denoted by $K_{t}$, has $t$ vertices, between any two of which there is a pair of arcs, one in each direction.

The connectivity of a connected digraph $\Gamma$ is the smallest possible size of a subset $W$ of $V \Gamma$ for which the induced digraph $\Gamma \backslash W$ is disconnected. By convention, the connectivity of a single vertex is 0 , and the connectivity of $K_{t}$ is $t-1$. A lobe of $\Gamma$ is a connected subgraph that is maximal subject to the condition that it has connectivity strictly greater than one. If $\Gamma$ has connectivity one, then the vertices $\alpha$ for which $\Gamma \backslash\{\alpha\}$ is disconnected are called the cut vertices of $\Gamma$.

A group acting on a digraph $\Gamma$ is said to be $k$-distance-transitive if, for any four vertices $\alpha_{1}, \alpha_{2}, \beta_{1}, \beta_{2} \in V \Gamma$ with $d\left(\alpha_{1}, \alpha_{2}\right)=d\left(\beta_{1}, \beta_{2}\right) \leq k$, there exists $g \in G$ such that $\alpha_{1}^{g}=\beta_{1}$ and $\alpha_{2}^{g}=\beta_{2}$, and is distance-transitive if it is $k$-distance-transitive for all positive integers $k$.

A digraph is distance-transitive if its automorphism group acts upon it distance-transitively. Note that a distance-transitive digraph $\Gamma$ is 1-distance transitive, and therefore its automorphism group maps any arc $(\alpha, \beta) \in A \Gamma$ to $(\beta, \alpha)$. Thus, between any two adjacent vertices in a distance-transitive digraph there are two arcs, one in each direction; where necessary the digraph can therefore be thought of as being an undirected graph.

The locally finite infinite distance-transitive graphs (and thus digraphs) were classified by Macpherson [8] and independently by Ivanov [7]. 
Theorem 1.2. ([8, Theorem 1.2]) An infinite locally finite digraph $\Gamma$ is distance-transitive if and only if it is a regular combinatorial tree, or $\Gamma$ has connectivity one, and for some integers $m \geq 2$ and $t \geq 3$ the lobes of $\Gamma$ are isomorphic to the complete digraph $K_{t}$, with each vertex in $\Gamma$ lying in $m$ lobes.

Adeleke and Neumann in [1, Remark 29.8] observe that if $G$ acts primitively on an infinite set $\Omega$ and has a non-trivial self-paired finite suborbit of size $m$ then $\Omega$ is countable, all the suborbits of $G$ are finite, $m \geq 2$, and if the suborbits are arranged in a non-decreasing sequence $1=m_{0} \leq m_{1} \leq \cdots$ then $m_{r} \leq(m-1) m_{r-1}$ for all sufficiently large $r$. They then ask whether the subdegree growth rates of infinite primitive groups that act distance-transitively on locally finite distance-transitive digraphs are extremal and conjecture there might exist a number $c$ which perhaps depends upon $G$, perhaps only on $m$, such that $m_{r} \leq c(m-2)^{r-1}$.

In fact, this approach is naive, as it does not consider the existence of a subdegree finite primitive group $G$, possessing at least two distinct subdegrees $m$ and $m^{\prime}$ such that infinitely many $\alpha$-suborbits have cardinality $m$, and infinitely many have cardinality $m^{\prime}$. Such subdegrees will henceforth be said to occur infinitely often. Indeed, if $G$ has at least two subdegrees, each occurring infinitely often, of which $m$ is the smallest, then under the above enumeration method $m_{r}=m$ for all sufficiently large $r$. Any subdegree of $G$ that is strictly larger than $m$ would therefore not be present in the subdegree sequence $\left(m_{r}\right)$.

In this paper we give examples of such groups, then define comprehensive methods for enumerating the subdegrees of subdegree finite infinite primitive groups and measuring their subdegree growth. Following this, we show that certain rates of subdegree growth determine the structure of the group.

\section{Examples and constructions}

A half-line $L$ of a digraph $\Gamma$ is a one-way infinite cycle-free path in $\Gamma$. The ends of $\Gamma$ are sets of half-lines, in which two half-lines $L_{1}$ and $L_{2}$ lie in the same end if and only if there exist an infinite number of pairwise-disjoint paths connecting a vertex in $L_{1}$ to a vertex in $L_{2}$. In fact, this definition is an equivalence relation on the set of half-lines of $\Gamma$, and the ends of $\Gamma$ are the equivalence classes of this relation.

It was noted in $[\mathbf{1 2}]$ that if $G$ is an infinite primitive permutation group possessing no infinite subdegree, then any two orbital digraphs of $G$ have the same ends. Thus we define the permutation-ends of $G$ to be the set of ends of an orbital digraph of $G$. It was also observed that all infinite locally finite primitive digraphs have one end, or they have $2^{\aleph_{0}}$ ends; thus any infinite subdegree finite primitive permutation group must have precisely one permutation-end, or $2^{\aleph_{0}}$ permutation-ends.

In this section we give examples of subdegree finite infinite primitive groups with exactly one permutation-end, and examples with an infinite number of permutation-ends. In both cases the groups in question possess at least two distinct subdegrees, both of which occur infinitely often.

It should be noted that the following examples may be used to construct several new examples of locally finite infinite primitive digraphs, which when taken to be undirected yield examples of locally finite infinite primitive graphs.

\subsection{Monster groups}

In [9], Ol'shanskiu shows that for every prime $p>10^{10}$, there is an infinite group in which all non-trivial proper subgroups are of order $p$. We propose to call such groups Tarski-Ol'shanskil monster $p$-groups. If $p$ is a prime number greater than $10^{10}$ and $T_{p}$ is such a group, fix any 
non-trivial proper subgroup $H \leq T_{p}$. Let $T_{p}$ act on the set of right cosets $\Omega:=\left\{H g \mid g \in T_{p}\right\}$ via

$$
(H g)^{g^{\prime}}=H g g^{\prime} .
$$

The kernel $K$ of this action is a normal subgroup of $T_{p}$ and is therefore trivial, so this action on $\Omega$ is faithful.

The stabiliser of the coset $H .1 \in \Omega$ is the group $H \leq T_{p}$. Since every subgroup of $T_{p}$ has order $p$, this group is a maximal subgroup of $T_{p}$. Furthermore, every element of the finite group $H$ has order $p$, so the orbits of $H$ acting on $\Omega \backslash\{H\}$ must all have size $p$. Hence $T_{p}$ acts primitively on $\Omega$, with all non-trivial suborbits finite of size $p$.

It was shown in [12] that any infinite primitive subdegree-finite permutation group possessing an orbital digraph with more than one end may be written as an amalgamated free product. In particular, such groups must have at least one element of infinite order. The group $T_{p}$ contains no element of infinite order, so every orbital digraph of this group must have precisely one end. Thus, $T_{p}$ is an example of a subdegree finite primitive group with one permutation-end, whose suborbits are all bounded above.

\subsection{Infinitely-ended constructions}

Let $\Lambda$ be any digraph with at least three vertices and connectivity strictly greater than one, and let $m$ be any cardinal number strictly greater than one. There is a digraph of connectivity one in which each lobe is isomorphic to $\Lambda$, and each vertex lies in precisely $m$ lobes. This digraph is unique up to isomorphism and we shall denote it by $\Gamma(m, \Lambda)$. Indeed, consider the following construction.

Let $\kappa$ be the cardinality of the set of vertices of $\Lambda$, and let $T$ be the bivalent tree in which vertices in one part of the natural bipartition of $T$ have valency $\kappa$, and the vertices in the other part have valency $m$. Colour vertices with valency $\kappa$ blue, and vertices with valency $m$ violet, and denote the set of blue vertices by $\mathfrak{B}$, and the set of violet vertices by $\mathfrak{W}$. Now take $\mathfrak{W}$ to be the vertex set of $\Gamma(m, \Lambda)$.

For each blue vertex $b$ of $T$, let $V_{b} \subset V \Gamma(m, \Lambda)$ be the set of vertices adjacent to $b$ in $T$, and choose any bijection

$$
\psi_{b}: V_{b} \rightarrow V \Lambda
$$

We then define the arc set $A \Gamma(m, \Lambda)$ thus:

$$
A \Gamma(m, \Lambda)=\bigcup_{b \in \mathfrak{B}}\left\{(\alpha, \beta) \in V_{b} \times V_{b} \mid\left(\psi_{b}(\alpha), \psi_{b}(\beta)\right) \in A \Lambda\right\} .
$$

It a simple exercise to check that the resulting digraph is well-defined and unique up to isomorphism.

Since $T$ and $\Lambda$ are connected, the digraph $\Gamma(m, \Lambda)$ must also be connected. Furthermore, if $b_{1}, b_{2} \in \mathfrak{B}$ are distinct then $\left|V_{b_{1}} \cap V_{b_{2}}\right| \leq 1$, and if $\gamma \in V_{b_{1}}$ and $\delta \in V_{b_{2}}$ and $\gamma, \delta \notin V_{b_{1}} \cap V_{b_{2}}$, then there is no arc in $\Gamma(m, \Lambda)$ between them. Whence $\Gamma(m, \Lambda)$ has connectivity one, and the lobes are isomorphic to $\Lambda$.

The digraph $\Gamma(m, \Lambda)$ was built from a tree, and any group acting on $\Gamma(m, \Lambda)$ has a natural action on that tree. This is a property of all digraphs with connectivity one. Indeed, suppose we are given a digraph with connectivity one. The bipartite digraph $T$ whose vertex set is the union of the set $V_{1}$ of cut vertices of $\Gamma$ and the set $V_{2}$ of lobes of $\Gamma$, in which $\alpha \in V_{1}$ is adjacent to $x \in V_{2}$ in $T$ if and only if $\alpha$ lies in $x$ in $\Gamma$, is in fact a tree, and is known as the block-cut-vertex tree of $\Gamma$. Note that if $\Gamma$ has connectivity one and block-cut-vertex tree $T$, then any group $G$ acting on $\Gamma$ has a natural action on $T$. 
Suppose we are given an integer $m \geq 2$, a locally finite primitive arc-transitive digraph $\Lambda$ with connectivity at least two, and an arc-transitive primitive non-regular group $H$ of automorphisms of $\Lambda$. We will construct a primitive and arc-transitive group of automorphisms $G$ of the digraph $\Gamma(m, \Lambda)$ such that the subgroup of Aut $\Lambda$ induced by $G_{\{\Lambda\}}$ is equal to $\bar{H}$, the closure of $H$ in Aut $\Lambda$ in the natural complete topology on Sym $V \Gamma$. This construction will provide a natural way of manufacturing large primitive groups from smaller ones.

A relational structure is a pair $(\Omega ; \Re)$, where $\mathfrak{R}$ is a set of relations on the set $\Omega$. Suppose we are given two relational structures $\left(\Omega_{1} ; \mathfrak{R}_{1}\right)$ and $\left(\Omega_{2} ; \mathfrak{R}_{2}\right)$, and a bijective map $\phi: \mathfrak{R}_{1} \rightarrow \mathfrak{R}_{2}$. A map $\varphi: \Omega_{1} \rightarrow \Omega_{2}$ is said to be an isomorphism between the relational structures $\left(\Omega_{1} ; \Re_{1}\right)$ and $\left(\Omega_{2} ; \mathfrak{R}_{2}\right)$ if and only if $\varphi(R)=\phi(R)$ for all relations $R \in \mathfrak{R}_{1}$, where $\varphi(R)$ denotes the set $\left\{\left(\varphi\left(\alpha_{1}\right), \ldots, \varphi\left(\alpha_{n}\right)\right) \mid\left(\alpha_{1}, \ldots, \alpha_{n}\right) \in R\right\}$.

If $G$ is a permutation group acting on a non-empty set $\Omega$, and $\Theta$ is an orbit of $G$ on $\Omega^{n}$, then it may be considered to be a relation of arity $n$ on $\Omega$. Following [2], we define $\mathfrak{R}_{G}$ to be the set of relations consisting of all orbits of $G$ on $\Omega^{n}$ where $n$ takes every value in the set of natural numbers. The canonical relational structure associated with $G$ is the relational structure $\left(\Omega ; \mathfrak{R}_{G}\right)$.

TheOREm 2.1. ([2, Theorem 2.6]) If $\Omega$ is a countably infinite set then a group $G$ of permutations of $\Omega$ is closed if and only if $G$ is the automorphism group of the canonical relational structure of $G$.

We begin by describing a relational structure that is based on the digraph $\Gamma(m, \Lambda)$. Let $\Gamma$ denote $\Gamma(m, \Lambda)$, and let $\mathfrak{R}$ be a set of relations on the set $V \Lambda$. Since all lobes of $\Gamma$ are isomorphic to $\Lambda$, we may, for each lobe $\Delta$ of $\Gamma$, choose a digraph isomorphism

$$
\varphi_{\Delta}: \Lambda \rightarrow \Delta \text {. }
$$

For each relation $R \in \Re$ define $R_{\Delta}:=\varphi_{\Delta}(R)$, where

$$
\varphi_{\Delta}(R):=\left\{\left(\varphi_{\Delta}\left(\alpha_{1}\right), \ldots, \varphi_{\Delta}\left(\alpha_{n}\right)\right) \mid\left(\alpha_{1}, \ldots, \alpha_{n}\right) \in R\right\} .
$$

Now define $\mathfrak{R}_{\Delta}:=\left\{R_{\Delta} \mid R \in \mathfrak{R}\right\}$, and observe that the relational structure $\left(V \Delta ; \mathfrak{R}_{\Delta}\right)$ is isomorphic to $(V \Lambda ; \mathfrak{R})$.

Using these relations, which are defined solely on the vertices of each lobe of $\Gamma$, it is possible to describe a set of relations defined on the whole of $V \Gamma$ thus: for each relation $R \in \mathfrak{R}$ let

$$
R_{\Gamma}:=\bigcup\left\{R_{\Delta} \mid \Delta \text { a lobe in } \Gamma\right\} .
$$

Now let $\mathfrak{S}:=\left\{R_{\Gamma} \mid R \in \mathfrak{R}\right\}$, and define $\Gamma(m, \Lambda, \mathfrak{R})$ to be the relational structure $(V \Gamma ;\{A \Gamma\} \cup$ $\mathfrak{S})$. This structure has many important properties, the most useful being

$$
\text { Aut } \Gamma(m, \Lambda, \mathfrak{R}) \leq \text { Aut } \Gamma(m, \Lambda) \text {. }
$$

Indeed, since the set $A \Gamma$ of $\operatorname{arcs}$ of $\Gamma$ is a relation in $\Gamma(m, \Lambda, \mathfrak{R})$, any automorphism of this relational structure must preserve $A \Gamma$ setwise, and is therefore an automorphism of $\Gamma$.

For each lobe $\Delta$ of $\Gamma(m, \Lambda)$ the relational structure $\left(V \Delta ; \mathfrak{R}_{\Delta}\right)$ is isomorphic to $(V \Lambda ; \mathfrak{R})$. Henceforth, such relational structures will be referred to as relational lobes of $\Gamma(m, \Lambda, \mathfrak{R})$.

Recall that we have been given a primitive and non-regular group $H$ that acts arc-transitively on $\Lambda$. The canonical set of relations $\mathfrak{R}_{H}$ consists of all orbits of $H$ on $V \Lambda^{n}$, where $n$ takes every value in the set of natural numbers. Let

$$
G:=\text { Aut } \Gamma\left(m, \Lambda, \Re_{H}\right) .
$$

We will show that the subgroup of Aut $\Lambda$ induced by $G_{\{\Lambda\}}$ is equal to $\bar{H}$, the closure of $H$ in Aut $\Lambda$. 

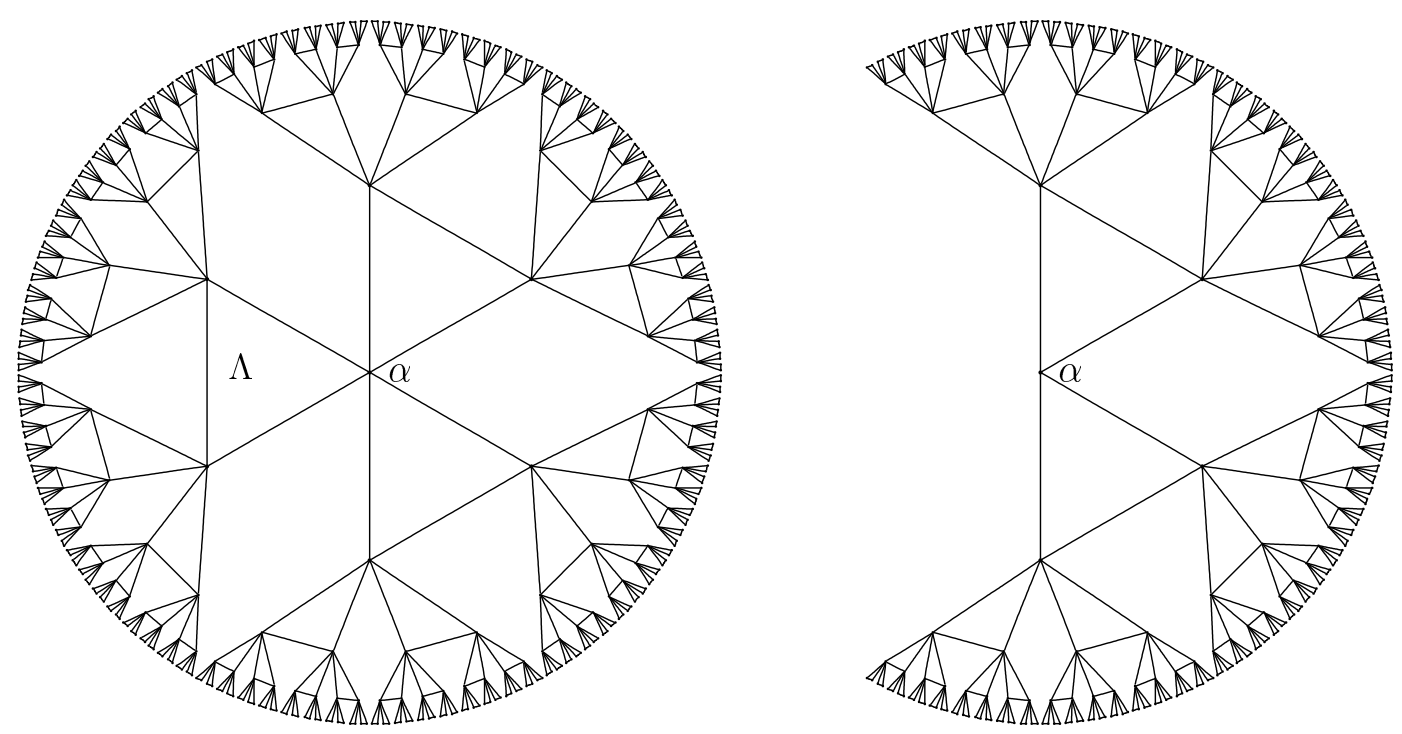

Figure 1. Digraphs $\Gamma$ (left) and $\Gamma_{\alpha}$ (right)

To clarify our exposition, we will, without loss of generality, assume that $\Lambda$ is a lobe in $\Gamma$. For each vertex $\alpha \in V \Lambda$ the digraph $\Gamma \backslash\{\alpha\}$ is not connected. One connected component of $\Gamma \backslash\{\alpha\}$ contains all vertices in $\Lambda \backslash\{\alpha\}$; the other connected components are disjoint from $\Lambda \backslash\{\alpha\}$. Define $\Gamma_{\alpha}^{\prime}$ to be the subgraph of $\Gamma$ consisting of all connected components of $\Gamma \backslash\{\alpha\}$ that are disjoint from $\Lambda \backslash\{\alpha\}$, and let $\Gamma_{\alpha}$ be the subgraph of $\Gamma$ induced by $V \Gamma_{\alpha}^{\prime} \cup\{\alpha\}$. This digraph has connectivity one, and for two vertices $\alpha, \beta \in V \Lambda$ the digraphs $\Gamma_{\alpha}$ and $\Gamma_{\beta}$ are isomorphic (see Figure 1).

We now describe a relational structure on each digraph $\Gamma_{\alpha}$ which will be used in the proof of the following lemma. Fix $\alpha \in V \Lambda$, and for each relation $R \in \mathfrak{R}$ let

$$
R_{\Gamma_{\alpha}}:=\bigcup\left\{R_{\Delta} \mid \Delta \text { a lobe in } \Gamma_{\alpha}\right\}
$$

and $\mathfrak{S}_{\alpha}:=\left\{R_{\Gamma_{\alpha}} \mid R \in \mathfrak{R}\right\}$. The latter is a set of relations on the set $V \Gamma_{\alpha}$, so we may define $\Sigma_{\alpha}$ to be the relational structure $\left(V \Gamma_{\alpha} ;\left\{A \Gamma_{\alpha}\right\} \cup \mathfrak{S}_{\alpha}\right)$, where $A \Gamma_{\alpha}$ is the set of arcs in $\Gamma_{\alpha}$.

For each $\gamma \in V \Lambda$, choose a digraph isomorphism $\phi_{\gamma}: \Gamma_{\alpha} \rightarrow \Gamma_{\gamma}$, with $\phi_{\alpha}$ the identity map. Now define $\mathfrak{S}_{\gamma}:=\phi_{\gamma}\left(\mathfrak{S}_{\alpha}\right)$, where $\phi_{\gamma}\left(\mathfrak{S}_{\alpha}\right)=\left\{\phi_{\gamma}(R) \mid R \in \mathfrak{S}_{\alpha}\right\}$, and let $\Sigma_{\gamma}$ be the relational structure $\left(V \Gamma_{\gamma} ;\left\{A \Gamma_{\gamma}\right\} \cup \mathfrak{S}_{\gamma}\right)$. The mapping $\phi_{\gamma}$ is thus an isomorphism between the relational structures $\Sigma_{\alpha}$ and $\Sigma_{\gamma}$. This observation will underpin the proof of our next lemma.

Lemma 2.2. Let $T$ be the block-cut-vertex tree of $\Gamma(m, \Lambda)$ and let $x$ be the vertex of $T$ corresponding to $\Lambda$. If $\alpha \in V \Lambda$ and $\beta \in V \Gamma$ lie in the same component of $T \backslash\{x\}$ then the subgroup of Aut $\Lambda$ induced by $G_{\alpha, \beta,\{\Lambda\}}$ is isomorphic to $\bar{H}_{\alpha}$.

Proof. Let $G^{\prime}$ be the subgroup of Aut $\Lambda$ induced by $G_{\alpha, \beta,\{\Lambda\}}$. It is clear that $G^{\prime} \leq$ Aut $\left(V \Lambda ; \mathfrak{R}_{H}\right)$. By Theorem 2.1 , Aut $\left(V \Lambda ; \mathfrak{R}_{H}\right)=\bar{H}$. Thus,

$$
G^{\prime} \leq \bar{H}_{\alpha} .
$$

Choose any automorphism $h \in \bar{H}_{\alpha}$. We will extend this to an automorphism $\sigma$ of $\Gamma\left(m, \Lambda, \Re_{H}\right)$ that fixes $\beta$. 
For each pair of vertices $\gamma_{1}, \gamma_{2} \in V \Lambda$ the relational structures $\Sigma_{\gamma_{1}}$ and $\Sigma_{\gamma_{2}}$ are isomorphic, so we may choose an isomorphism

$$
\phi_{\left(\gamma_{1}, \gamma_{2}\right)}: \Sigma_{\gamma_{1}} \rightarrow \Sigma_{\gamma_{2}}
$$

which is equal to the identity map when $\gamma_{1}=\gamma_{2}$. We now construct a mapping $\sigma$ : $\Gamma\left(m, \Lambda, \mathfrak{R}_{H}\right) \rightarrow \Gamma\left(m, \Lambda, \mathfrak{R}_{H}\right)$ as follows. For each $\delta \in V \Gamma$ there exists a unique vertex $\gamma \in V \Lambda$ such that $\delta \in \Sigma_{\gamma}$, so set

$$
\delta^{\sigma}:=\delta^{\phi}\left(\gamma, \gamma^{h}\right) .
$$

It is simple to check this is a well-defined automorphism of $\Gamma\left(m, \Lambda, \mathfrak{R}_{H}\right)$. Since $\alpha$ and $\beta$ lie in the same component of $T \backslash\{x\}$, the vertex $\alpha$ must be the unique element of $V \Lambda$ such that $\beta \in \Sigma_{\alpha}$. Hence $\beta^{\sigma}=\beta$, so $G^{\prime}=\bar{H}_{\alpha}$.

Before introducing our next result, a little notation is necessary. Since there is a unique geodesic between any two vertices $\alpha$ and $\beta$ in a tree $T$, we shall denote the geodesic between $\alpha$ and $\beta$ that includes both vertices by $[\alpha, \beta]_{T}$; if we wish to exclude $\beta$ then we instead write $[\alpha, \beta)_{T}$.

THEOREM 2.3. Suppose $H$ is a primitive non-regular arc-transitive group of automorphisms of a locally finite digraph $\Lambda$ with at least three vertices and connectivity strictly greater than one. If $m \geq 2$ then the automorphism group Aut $\Gamma\left(m, \Lambda, \mathfrak{R}_{H}\right)$ acts primitively and arc-transitively on the digraph $\Gamma(m, \Lambda)$. Furthermore, the group induced by its action on $V \Lambda$ is $\bar{H}$, the closure of $H$ in Aut $\Lambda$.

Proof. We begin by showing that the subgroup of Aut $\Lambda$ that is induced by Aut $\Gamma\left(m, \Lambda, \mathfrak{R}_{H}\right)$ on $V \Lambda$ is $\bar{H}$. Let $G=$ Aut $\Gamma\left(m, \Lambda, \mathfrak{R}_{H}\right)$ and let $G^{\prime}$ be the subgroup of Aut $\Lambda$ induced by the action of $G_{\{\Lambda\}}$ on $V \Lambda$. By construction, $G \leq$ Aut $\Gamma(m, \Lambda)$. Fix $\alpha \in V \Lambda$, and note that by applying Lemma 2.2 with $\beta=\alpha$ we have $G_{\alpha}^{\prime}=\bar{H}_{\alpha}$. Furthermore, $G^{\prime} \leq$ Aut $\left(V \Lambda, \mathfrak{R}_{H}\right)=\bar{H}$, so

$$
\bar{H}_{\alpha} \leq G^{\prime} \leq \bar{H} .
$$

Our choice of $\alpha$ was arbitrary, so we may choose a vertex $\gamma \in V \Lambda$ that is distinct from $\alpha$ and note that

$$
\bar{H}_{\gamma} \leq G^{\prime} \leq \bar{H} .
$$

Since $H$ acts primitively and non-regularly on $V \Lambda$, the group $\bar{H}_{\gamma}$ does not fix $\alpha$; whence $G^{\prime}$ does not fix $\alpha$. Thus

$$
\bar{H}_{\alpha}<G^{\prime} \leq \bar{H} .
$$

Since $H$ acts primitively on $V \Lambda$ the same must be true of $\bar{H}$; therefore $\bar{H}_{\alpha}$ is a maximal subgroup of $\bar{H}$. Consequently $G^{\prime}=\bar{H}$.

It remains to show that $G$ acts primitively and arc-transitively on the digraph $\Gamma(m, \Lambda)$. By construction $G$ acts transitively on the relational lobes of $\Gamma\left(m, \Lambda, \mathfrak{R}_{H}\right)$, and therefore acts transitively on the set of lobes of the digraph $\Gamma(m, \Lambda)$.

The action of $G$ on the vertices of $\Gamma$ is transitive. Indeed, suppose $\delta$ is any vertex in $V \Gamma$. Then there exists a lobe $\Delta$ of $\Gamma$ containing $\delta$. Choose $g \in G$ such that $\Delta^{g}=\Lambda$. Since $G^{\prime}=\bar{H}$, and $H$ acts transitively on the vertices of $\Lambda$, there exists an automorphism $h \in G_{\{\Lambda\}}$ such that $\delta^{g h}=\alpha$.

Furthermore, the stabiliser $G_{\alpha}$ transitively permutes the lobes of $\Gamma$ that contain $\alpha$. For, suppose $\Lambda^{\prime}$ is a lobe of $\Gamma$ containing $\alpha$. Then there is an automorphism $g \in G$ such that 
$\Lambda^{g}=\Lambda^{\prime}$. We again observe that since $G^{\prime}=\bar{H}$, and $H$ acts transitively on the vertices of $\Lambda$, there exists an automorphism $h \in G_{\{\Lambda\}}$ such that $\alpha^{h}=\alpha^{g^{-1}}$. Thus $\Lambda^{h g}=\Lambda^{\prime}$ and $\alpha^{h g}=\alpha$.

Since $H$ acts vertex- and arc-transitively on $\Lambda$, and $G_{\alpha}$ transitively permutes the lobes of $\Gamma$ that contain $\alpha$, the group $G$ must be arc-transitive on $\Gamma$.

Finally, let $\rho$ be a non-trivial $G$-congruence on $V \Gamma$. Let $T$ be the block-cut-vertex tree of $\Gamma$. Choose $\beta \in \rho(\alpha) \backslash\{\alpha\}$ of minimal distance in $T$ from $\alpha$. We claim $\alpha$ and $\beta$ lie in a common lobe of $\Gamma$. Indeed, suppose this is not the case. Let $x$ be the vertex adjacent to $\beta$ in the geodesic $[\alpha, \beta]_{T}$ between $\alpha$ and $\beta$, and let $\gamma$ be the vertex adjacent to $x$ in $[\alpha, \beta)_{T}$. The vertex $x$ corresponds to a lobe $\Lambda^{\prime}$ of $\Gamma$, which, since $\alpha$ and $\beta$ lie in distinct lobes, is not equal to $\Lambda$. Because $G$ acts transitively on the lobes of $\Gamma$, the group induced on $V \Lambda^{\prime}$ by $G_{\alpha, \gamma,\left\{\Lambda^{\prime}\right\}}$ is isomorphic to $\bar{H}_{\alpha}$ acting on $V \Lambda$. Since $\bar{H}$ is primitive and non-regular on $V \Lambda$, there exists $g \in G_{\alpha, \gamma,\left\{\Lambda^{\prime}\right\}}$ such that $\beta^{g} \neq \beta$. Thus $\beta, \beta^{g} \in \rho(\alpha)$ lie in a common lobe, and our claim is established. So, without loss of generality, we may assume $\alpha$ and $\beta$ lie in $\Lambda$. Now $\rho$ induces a $\bar{H}$-congruence on $V \Lambda$, so we must have $V \Lambda \subseteq \rho(\alpha)$. Furthermore, $G_{\alpha}$ acts transitively on the lobes of $\Gamma$ that contain $\alpha$, so $\rho$ is the universal relation. Hence $G$ acts primitively on $\Gamma$.

If $H$ is a primitive and non-regular group of permutations of a set $\Omega$, with $|\Omega| \geq 3$, possessing an orbital digraph $\Lambda$ with connectivity strictly greater than one, then the primitive group $G$ constructed above will be called the $m$-fold graph product of $H$, and will be denoted by $G(m, H)$. By the above theorem, this group acts primitively on the vertex set of $\Gamma(m, \Lambda)$. Since this digraph has infinitely many ends, and is an orbital digraph of $G(m, \Lambda)$, all orbital digraphs of $G$ will have infinitely many ends.

EXAMPLE 1. Let $m$ be a finite cardinal number with $m \geq 2$, let $p$ be a prime number with $p>10^{10}$, and let $T_{p}$ be a Tarski-Ol'shanskiu monster $p$-group. Recall this group acts primitively on its coset space $\Omega$. Let $\Lambda$ be a non-diagonal orbital digraph of $T_{p}$. Then $\Lambda$ is a one-ended primitive digraph, and therefore has connectivity strictly greater than one.

By Theorem 2.3, the group $G\left(m, T_{p}\right)$ acts primitively on $\Gamma(m, \Lambda)$, and has infinitely many distinct subdegrees, each occurring infinitely often. In fact, for all $\alpha \in V \Gamma$, the $\alpha$-subdegrees of $G\left(m, T_{p}\right)$ are $m(m-1)^{r-1} p^{r}$ for $r \geq 1$. For each positive integer $r$ there are infinitely many $\alpha$-suborbits of $G\left(m, T_{p}\right)$ with cardinality $m(m-1)^{r-1} p^{r}$.

\subsection{One-ended constructions}

The following result allows one to construct infinitely many examples of subdegree finite primitive groups with one permutation-end.

THEOREM 2.4. If $G$ is a subdegree finite primitive group of permutations of an infinite set $\Omega$ and $m \geq 2$, then the wreath product $G \operatorname{Wr} \operatorname{Sym}(m)$ under the product action is a subdegree finite primitive group of permutations of $\Omega^{m}$ with one permutation-end.

This result may be deduced from the following lemmas. Fix a group $G$ acting primitively on an infinite set $\Omega$, possessing a finite suborbit whose pair is also finite. Let $\Gamma$ be an orbital digraph of $G$ on $\Omega$, and define $H:=G \mathrm{Wr} \operatorname{Sym}(m)$. It is well known that $H$ acts primitively on the set $\Omega^{m}$ under the product action; see, for example [4, Lemma 2.7A]. Fix $\alpha, \beta \in \Omega$ such that $\alpha$ and $\beta$ are adjacent in $\Gamma$, with $(\alpha, \beta) \in A \Gamma$. Let $\underline{\alpha}:=(\alpha, \ldots, \alpha) \in \Omega^{m}$ and $\underline{\beta}:=(\beta, \alpha, \ldots, \alpha) \in \Omega^{m}$, and define

$$
\Lambda:=\left(\Omega^{m},(\underline{\alpha}, \underline{\beta})^{H}\right),
$$


where $H$ acts on $\Omega^{m}$ via the product action.

Lemma 2.5. The vertex $\left(\gamma_{1}, \ldots, \gamma_{m}\right)$ is adjacent to $\underline{\alpha}$ in $\Lambda$ if and only if

$$
\sum_{i=1}^{m} d_{\Gamma}\left(\alpha, \gamma_{i}\right)=1
$$

Proof. Let $\gamma:=\left(\gamma_{1}, \ldots, \gamma_{m}\right) \in V \Lambda$. Since $\Lambda$ is an orbital digraph of $H$ on $\Omega^{m}$, it is arc transitive. Therefore $\gamma$ lies in the sphere $S_{1}(\underline{\alpha}, \Lambda)$ if and only if there exists $g=\left(g_{1}, \ldots, g_{m}\right) \sigma \in$ $H$ such that $(\underline{\alpha}, \beta)^{g^{-}}$is equal to $(\underline{\alpha}, \underline{\gamma})$ or $(\underline{\gamma}, \underline{\alpha})$. If $(\underline{\alpha}, \beta)^{g}=(\underline{\alpha}, \underline{\gamma})$ then $g \in H_{\underline{\alpha}}$ and so $g_{1}, \ldots, g_{m} \in G_{\alpha}$. Thus $\underline{\gamma}=\underline{\beta}^{g}=\left(\beta^{g_{1}}, \bar{\alpha}, \ldots, \alpha\right)^{\sigma}$, and therefore $\sum_{i=1}^{m} d_{\Gamma}\left(\alpha, \gamma_{i}\right)=d_{\Gamma}\left(\alpha, \beta^{g_{1}}\right)=$ 1. Otherwise, if $(\underline{\alpha}, \beta)^{g}=(\underline{\gamma}, \underline{\alpha})$ then $\underline{\beta}^{g}=\underline{\alpha}$, so $g_{2}, \ldots, g_{m} \in G_{\alpha}$ but $\beta^{g_{1}}=\alpha$. Thus $\underline{\gamma}=\underline{\alpha}^{g}=$ $\left(\alpha^{g_{1}}, \alpha, \ldots, \alpha\right)^{\sigma}$ and $\sum_{i=1}^{m} \bar{d}_{\Gamma}\left(\alpha, \gamma_{i}\right)=\bar{d}_{\Gamma}\left(\alpha, \alpha^{g_{1}}\right)=1$.

Conversely, suppose $\gamma=\left(\gamma_{1}, \ldots, \gamma_{m}\right) \in V \Lambda$ and $\sum_{i=1}^{m} d_{\Gamma}\left(\alpha, \gamma_{i}\right)=1$. Then there exists a unique value of $i$ such that $d_{\Gamma}\left(\alpha, \gamma_{i}\right)=1$ and $\gamma_{j}=\alpha$ for all $j \neq i$. Thus, there exists $g_{1} \in G$ such that $(\alpha, \beta)^{g_{1}}$ is equal to $\left(\alpha, \gamma_{i}\right)$ or $\left(\gamma_{i}, \alpha\right)$. Let $\sigma \in \operatorname{Sym}(m)$ be the 2-cycle $(1 i)$ and set $g:=\left(g_{1}, 1, \ldots, 1\right) \sigma \in H$. If $(\alpha, \beta)^{g_{1}}=\left(\alpha, \gamma_{i}\right)$ then $g \in H_{\alpha}$ and $\beta^{g}=\left(\beta^{g_{i}}, \alpha, \ldots, \alpha\right)^{\sigma}=$ $\left(\gamma_{i}, \alpha, \ldots, \alpha\right)^{\sigma}=\gamma$, and therefore $d_{\Lambda}(\underline{\alpha}, \gamma)=1$. On the other hand, if $(\alpha, \beta)^{g_{1}}=\left(\gamma_{i}, \alpha\right)$ then $\underline{\beta}^{g}=\underline{\alpha}$ and $\underline{\alpha}^{g}=\left(\alpha^{g_{1}}, \alpha, \ldots, \alpha\right)^{\sigma}=\left(\gamma_{i}, \bar{\alpha}, \ldots, \alpha\right)^{\sigma}=\underline{\gamma}$, so again $d_{\Lambda}(\underline{\alpha}, \underline{\gamma})=1$. We have thus shown $\left(\gamma_{1}, \ldots, \gamma_{m}\right) \in S_{1}((\alpha, \ldots, \alpha), \Lambda)$ if and only if $\sum_{i=1}^{m} d_{\Gamma}\left(\alpha, \gamma_{i}\right)=\overline{1}$. Since $\Lambda$ is vertextransitive the hypothesis holds for any vertex $\left(\delta_{1}, \ldots, \delta_{m}\right) \in V \Lambda$.

Lemma 2.6. The vertex $\left(\gamma_{1}, \ldots, \gamma_{m}\right)$ lies in $S_{r}\left(\left(\delta_{1}, \ldots, \delta_{m}\right), \Lambda\right)$ if and only if

$$
\sum_{i=1}^{m} d_{\Gamma}\left(\delta_{i}, \gamma_{i}\right)=r .
$$

Proof. We proceed by induction. Since $H$ is vertex-transitive, the hypothesis holds when $r=$ 1 by Lemma 2.5. Fix $\underline{\delta}=\left(\delta_{1}, \ldots, \delta_{m}\right) \in V \Lambda$ and $k>1$ and suppose the hypothesis is true for all $r \leq k$. Choose $\underline{\gamma}=\left(\gamma_{1}, \ldots, \gamma_{m}\right) \in V \Lambda$ with $\sum_{i=1}^{m} d_{\Gamma}\left(\delta_{i}, \gamma_{i}\right)=k+1$. Since $\sum_{i=1}^{m} d_{\Gamma}\left(\delta_{i}, \gamma_{i}\right)>2$ there exists $j$ for which one may choose $\gamma_{j}^{\prime} \in S_{1}\left(\gamma_{j}, \Gamma\right)$ such that $d_{\Gamma}\left(\delta_{j}, \gamma_{j}^{\prime}\right)=d_{\Gamma}\left(\delta_{j}, \gamma_{j}\right)-1$. Let $\gamma_{i}^{\prime}:=\gamma_{i}$ for all $i \neq j$ and put $\gamma^{\prime}:=\left(\gamma_{1}^{\prime}, \ldots, \gamma_{m}^{\prime}\right)$. Now $\sum_{i=1}^{m} d_{\Gamma}\left(\delta_{i}, \gamma_{i}^{\prime}\right)=k$, so by assumption, $\underline{\gamma}^{\prime} \in S_{k}(\underline{\delta}, \Lambda)$; furthermore, $\left.d_{\Lambda} \overline{(\underline{\gamma}}, \gamma^{\prime}\right)=1$, so $\gamma \in S_{k-1}(\underline{\delta}, \Lambda) \cup S_{k}(\underline{\delta}, \Lambda) \cup S_{k+1}(\underline{\delta}, \Lambda)$. If $\underline{\gamma}$ lies in $\bar{S}_{k-1}(\underline{\delta}, \underline{\Lambda})$ or $S_{k}(\underline{\delta}, \Lambda)$ then by assumption $\sum_{i=1}^{m} d_{\Gamma}\left(\delta_{i}, \gamma_{i}\right)$ is equal to $k-1$ or $k$ respectively; since this is not the case, we must have $\gamma \in S_{k+1}(\underline{\delta}, \Lambda)$.

Conversely, suppose $\underline{\gamma}=\left(\gamma_{1}, \ldots, \gamma_{m}\right) \in S_{k+1}(\underline{\delta}, \underline{\Lambda})$. Since $d_{\Lambda}(\underline{\delta}, \underline{\gamma})>k$ we have $\sum_{i=1}^{m} d_{\Gamma}\left(\delta_{i}, \gamma_{i}\right) \geq$ $k+1$ by the induction hypothesis. Since $\Lambda$ is connected, there exists $\gamma^{\prime}=\left(\gamma_{1}^{\prime}, \ldots, \gamma_{m}^{\prime}\right) \in$ $S_{k}(\underline{\delta}, \Lambda) \cap S_{1}(\underline{\gamma}, \Lambda)$. Now $\sum_{i=1}^{m} d_{\Gamma}\left(\delta_{i}, \gamma_{i}^{\prime}\right)=k$ and $\sum_{i=1}^{m} d_{\Gamma}\left(\gamma_{i}, \gamma_{i}^{\prime}\right)=1$. For each $i$ we have $d_{\Gamma}\left(\delta_{i}, \gamma_{i}\right) \leq d_{\Gamma}\left(\delta_{i}, \gamma_{i}^{\prime}\right)+d_{\Gamma}\left(\gamma_{i}^{\prime}, \gamma_{i}\right)$. Whence, $\sum_{i=1}^{m} d_{\Gamma}\left(\delta_{i}, \gamma_{i}\right) \leq \sum_{i=1}^{m}\left(d_{\Gamma}\left(\delta_{i}, \gamma_{i}^{\prime}\right)+d_{\Gamma}\left(\gamma_{i}^{\prime}, \gamma_{i}\right)\right)=$ $k+1$. Hence $\sum_{i=1}^{m} d_{\Gamma}\left(\delta_{i}, \gamma_{i}\right)=k+1$.

LEMma 2.7. The digraph $\Lambda$ is infinite, primitive, locally finite and arc-transitive, with one end.

Proof. The group $H$ acts primitively on $V \Lambda$, so the digraph $\Lambda$ is primitive. It is infinite because $V \Lambda=\Omega^{m}$ is infinite, and is locally finite by Lemma 2.5; furthermore, since $\Lambda$ is an orbital digraph of $H$ on $\Omega^{m}$, it is arc-transitive. 
It remains to prove that $\Lambda$ has one end. We will show, for all $r \geq 1$, given any pair of vertices $\underline{\gamma}, \underline{\delta} \in S_{r+1}(\underline{\alpha}, \Lambda)$, there is a path connecting $\underline{\gamma}$ to $\underline{\delta}$ that is not contained in the ball $B_{r}(\underline{\alpha}, \Lambda)$, where $B_{r}(\underline{\alpha}, \Lambda)$ is the set of all vertices in $\Lambda$ whose distance from $\underline{\alpha}$ is at most $r$. From this, we may deduce there is no finite subgraph of $\Lambda$ that one may remove to leave at least two disjoint infinite connected components. Whence, $\Lambda$ has precisely one end.

We begin by observing that, given any two vertices $\left(\gamma_{1}, \ldots, \gamma_{m}\right)$ and $\left(\delta_{1}, \ldots, \delta_{m}\right)$ in $V \Lambda$, for any path in $\Gamma$ between $\gamma_{1}$ and $\delta_{1}$ there exists a corresponding path in $\Lambda$ between $\left(\gamma_{1}, \gamma_{2}, \ldots, \gamma_{m}\right)$ and $\left(\delta_{1}, \gamma_{2}, \ldots, \gamma_{m}\right)$. Indeed, by Lemma 2.5 , if $\xi$ lies on the path in $\Gamma$ between $\gamma_{1}$ and $\delta_{1}$, then $\left(\xi, \gamma_{2}, \ldots, \gamma_{m}\right)$ lies on the corresponding path in $\Lambda$ between $\left(\gamma_{1}, \gamma_{2}, \ldots, \gamma_{m}\right)$ and $\left(\delta_{1}, \gamma_{2}, \ldots, \gamma_{m}\right)$. This observation can also be made for paths between $\gamma_{i}$ and $\delta_{i}$ for all $i$ satisfying $1 \leq i \leq m$.

Fix $r \geq 1$ and two distinct vertices $\underline{\gamma}=\left(\gamma_{1}, \ldots, \gamma_{m}\right)$ and $\underline{\delta}=\left(\delta_{1}, \ldots, \delta_{m}\right)$ in $S_{r+1}(\underline{\alpha}, \Lambda)$. Let $B_{r}:=B_{r}(\underline{\alpha}, \Lambda)$. We will describe four vertices $\underline{\xi}_{1}, \ldots, \underline{\xi_{4}} \in V \Lambda \backslash B_{r}$ such that there exist paths in $\Lambda$ between $\underline{\gamma}$ and $\underline{\xi_{1}}$; between $\underline{\delta}$ and $\underline{\xi_{4}}$; and between $\underline{\xi_{i}}$ and $\underline{\xi_{i+1}}$ for $1 \leq i<4$ that are all

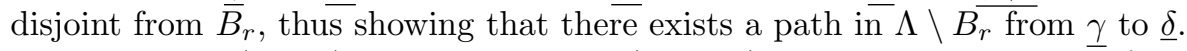

Let $d_{1}:=d_{\Gamma}\left(\alpha_{1}, \delta_{1}\right)-1$ and $d_{m}:=d_{\Gamma}\left(\alpha_{m}, \gamma_{m}\right)-1$. Choose a vertex $\gamma_{m}^{\prime} \in V \Gamma \backslash B_{r}\left(\alpha_{m}, \Gamma\right)$ such that there is a path in $\Gamma$ between $\gamma_{m}$ and $\gamma_{m}^{\prime}$ that is disjoint from $B_{d_{m}}\left(\alpha_{m}, \Gamma\right)$. Similarly, choose a vertex $\delta_{1}^{\prime} \in V \Gamma \backslash B_{r}\left(\alpha_{1}, \Gamma\right)$ such that there is a path in $\Gamma$ between $\delta_{1}$ and $\delta_{1}^{\prime}$ that is disjoint from $B_{d_{1}}\left(\alpha_{1}, \Gamma\right)$. Now define

$$
\begin{aligned}
& \underline{\xi_{1}}:=\left(\gamma_{1}, \gamma_{2}, \ldots, \gamma_{m-1}, \gamma_{m}^{\prime}\right) \\
& \underline{\underline{\xi_{2}}}:=\left(\delta_{1}, \delta_{2}, \ldots, \delta_{m-1}, \gamma_{m}^{\prime}\right) \\
& \underline{\underline{\xi_{3}}}:=\left(\delta_{1}^{\prime}, \delta_{2}, \ldots, \delta_{m-1}, \gamma_{m}^{\prime}\right) \\
& \underline{\xi_{4}}:=\left(\delta_{1}^{\prime}, \delta_{2}, \ldots, \delta_{m-1}, \delta_{m}\right) .
\end{aligned}
$$

The path in $\Gamma$ between $\gamma_{m}$ and $\gamma_{m}^{\prime}$ that is disjoint from $B_{d_{m}}\left(\alpha_{m}, \Gamma\right)$ corresponds to a path in $\Lambda$ between the vertices $\left(\gamma_{1}, \ldots, \gamma_{m-1}, \gamma_{m}\right)$ and $\left(\gamma_{1}, \ldots, \gamma_{m-1}, \gamma_{m}^{\prime}\right)$ which, by Lemma 2.6 , is disjoint from $B_{r}$. Hence, there exists a path in $\Lambda \backslash B_{r}$ between $\underline{\gamma}$ and $\underline{\xi_{1}}$. A similar argument shows that there exists a path in $\Lambda \backslash B_{r}$ between $\underline{\delta}$ and $\xi_{4}$.

Finally, observe that any vertex $\underline{\chi}=\left(\chi_{1}, \ldots, \chi_{m}\right) \in \overline{V \Lambda}$ satisfying $\chi_{i} \in V \Gamma \backslash B_{r}\left(\alpha_{i}, \Gamma\right)$ for some $i$ does not lie in $B_{r}$ by Lemma 2.6 . Therefore, there exist paths in $\Lambda \backslash B_{r}$ between $\underline{\xi_{1}}$ and $\underline{\xi_{2}}$; between $\underline{\xi_{2}}$ and $\underline{\xi_{3}}$; and between $\underline{\xi_{3}}$ and $\underline{\xi_{4}}$. Hence, $\Lambda$ has precisely one end.

Thus the proof of Theorem 2.4 is complete.

EXAMPLE 2. Let $p$ be a prime number greater than $10^{10}$ and let $T_{p}$ be a TarskiOl'shanskil monster $p$-group. Recall that this group acts primitively on its coset space $\Omega$. Let $G:=T_{p} \mathrm{Wr} \operatorname{Sym}(2)$. This group acts primitively on the set $\Omega^{2}$ by Theorem 2.4 , and has precisely three non-trivial subdegrees, $2 p, p^{2}$ and $2 p^{2}$, each occurring infinitely often.

EXAmple 3. The orbital digraphs of the group $G\left(m, T_{p}\right) \mathrm{Wr}$ Sym (2) have precisely one end. Furthermore, this group has infinitely many distinct subdegrees, each occurring infinitely often. 


\section{Enumeration and growth}

\subsection{The upper and lower subdegree sequences}

As the above examples show, the concept of subdegree growth requires re-examining. We begin by clarifying some terminology. Let $G$ act primitively on an infinite set $\Omega$, possessing a finite suborbit whose pair is also finite. Then every suborbit of $G$ is finite. Fix $\alpha \in \Omega$.

The set of subdegrees of $G$ is defined to be the set whose elements are the cardinalities of its $\alpha$-suborbits. The multiset of subdegrees of $G$ is a function $\mu$ from the set of subdegrees to the set of countable (finite and countably infinite) cardinal numbers, defined as follows. If $m$ is any subdegree of $G$ then $\mu(m)$ is the number of $\alpha$-suborbits with cardinality $m$. Having defined this multiset formally, we shall speak of it informally as a set of elements of the set of subdegrees, in which some elements occur with multiplicity greater than one.

For our given group $G$, there exists a minimal ordinal number $\kappa$ such that one may enumerate all elements of the multiset of subdegrees as a monotonic increasing sequence $\left(m_{\gamma}\right)$ for $\gamma<\kappa$. This sequence $\left(m_{\gamma}\right)$ is called the subdegree sequence of $G$, and the ordinal number $\kappa$ is called the height of $G$. The precise definition of the subdegree sequence of $G$ is now hopefully clear: $\left(m_{\gamma}\right)$ is a non-decreasing sequence of elements of the set of subdegrees, in which each subdegree $m$ appears in the sequence with multiplicity $\mu(m)$.

By Theorem 1.1, the set of subdegrees of $G$ is a finite or countably infinite set. Since each entry occurs with multiplicity one, we may enumerate all its elements $M_{1}<M_{2}<\cdots$. The sequence $\left(M_{r}\right)$ is called the upper subdegree sequence of $G$. The lower subdegree sequence of $G$ is the sequence $\left(m_{r}\right)_{r<\omega}$. Note that both the upper and lower subdegree sequences are subsequences of the subdegree sequence of $G$, and are indexed by the natural numbers.

LEMMA 3.1. If $G$ is a subdegree finite primitive group with height $h$ then

$$
\omega \leq h \leq \omega^{2}
$$

Proof. Let $X$ and $Y$ be the set and multiset of subdegrees of $G$ respectively. Since $G$ acts on an infinite set, and all subdegrees of $G$ are finite, $Y$ is infinite, so the height of $G$ is at least $\omega$.

Each subdegree in $X$ occurs with multiplicity at most $\aleph_{0}$ in $Y$, so the subdegree sequence can be enumerated in a non-decreasing sequence of length at most $\omega^{2}$.

Subdegree growth is similar in many ways to the growth of connected locally finite infinite digraphs, an area of research that is already well-establish. Following [14], if $t_{1} \leq t_{2} \leq t_{3} \leq \cdots$. is a sequence of positive real numbers, we define the concept of growth as follows. If there exist positive real numbers $c_{1}, c_{2}$ and $d \geq 1$ such that

$$
c_{1} r^{d} \leq t_{r} \leq c_{2} r^{d}
$$

we say that the sequence has polynomial growth of degree $d$. A sequence exhibiting polynomial growth of degree 0 is often called bounded. The growth is subexponential if, for all $a>1$,

$$
\liminf _{r \rightarrow \infty} \frac{t_{r}}{a^{r}}=0
$$

it is exponential if there exist constants $a, b>1$ such that $\liminf _{r \rightarrow \infty} t_{r} / a^{r}$ and $\limsup _{r \rightarrow \infty} t_{r} / b^{r}$ are non-zero and finite. The growth is said to be super exponential if $\liminf _{r \rightarrow \infty} t_{r} / a^{r}$ is infinite for all $a>1$.

The growth of an infinite locally finite vertex-transitive connected digraph $\Gamma$ is the growth of the sequence $\left(\left|B_{r}(\alpha, \Gamma)\right|\right)$. A great deal of work has been done in this area. It was shown in 
[6] that it is precisely the two-ended digraphs that have growth of degree one. Furthermore, Trofimov has shown in [13] that if a locally finite digraph has polynomial growth then it is not primitive. All locally finite infinitely-ended vertex-transitive digraphs have exponential sphere growth, while it is noted in [14] that one-ended digraphs exhibit all possible rates of growth of degree at least 2, except super exponential.

By examining the growth of both the lower and upper subdegree sequences, one may obtain similar results pertaining to subdegree growth rates. It is natural to consider the subdegree growth of primitive groups in this way. Indeed, the intuitive but flawed approach taken by Adeleke and Neumann in [1, Remark 29.8] only considered the existence of primitive groups with height $\omega$. For such groups, the lower subdegree sequence is equal to the subdegree sequence (although it is not necessarily equal to the upper subdegree sequence), so the two approaches are equivalent in this case.

For groups with height $\omega$, the growth of the upper subdegree sequence is of secondary importance, as all relevant subdegree information can be found in the lower subdegree sequence. However, this is not the case for groups with height strictly greater than $\omega$.

LEMma 3.2. If an infinite primitive group $G$ whose subdegrees are all finite does not have height $\omega$, then the lower subdegree sequence is always bounded.

Proof. Suppose $G$ has height $h>\omega$, and let $\left(m_{\gamma}\right)$ be the subdegree sequence of $G$. Then there are finite constants $c_{1}:=1$ and $c_{2}:=m_{\omega}$ such that $c_{1} \leq m_{r} \leq c_{2}$ for all $r<\omega$.

The growth of the upper subdegree sequence will be used only to differentiate between the subdegree growth of groups exhibiting a bounded lower subdegree sequence; thus, when referring to the subdegree growth of a group, unless otherwise stated, it is the lower subdegree growth to which we are referring.

The constructions detailed in this paper may be used to create myriad examples of groups with exponential, subexponential and polynomial growth, several of which are given below. The final example illustrates how they may also be used to show that the above list of possible rates of growth is not exhaustive.

ExAmple 4. Fix $m \geq 2$ and $t \geq 3$, and let $G:=\operatorname{Aut~} \Gamma\left(m, K_{t+1}\right)$. The group $G$ acts distance-transitively on $V \Gamma$, and has height $\omega$. The upper and lower subdegree sequences of $G$ are equal, with $M_{r}=m_{r}=m(m-1)^{r-1} t^{r}$, for all $r \in \mathbb{N}$.

EXAMPLE 5. If $p$ is prime, and $p>10^{10}$, then the group $G\left(m, T_{p}\right)$ has a bounded lower subdegree sequence, with $m_{r}=m p$ for all integers $r \geq 1$. However, $M_{r}=m(m-1)^{r-1} p^{r}$ for all $r \geq 1$. Intuitively, while this group exhibits slow subdegree growth when compared to groups with non-bounded lower subdegree sequences, when instead it is compared with other groups possessing bounded lower subdegree sequences its subdegree growth is extremely fast.

ExAmple 6. Given a prime integer $p$ with $p>10^{10}$, both $T_{p}$ and the wreath product $T_{p}$ Wr Sym (2) have bounded upper and lower subdegree sequences. The subdegree growth of these groups is therefore, intuitively, very slow. Indeed, $T_{p}$ provides a lower bound on both upper and lower subdegree growth rates.

Example 7. Fix integers $m, t \geq 2$ and define $\Gamma:=\Gamma\left(m, K_{t+1}\right)$. Let $G:=$ Aut $\Gamma$ and let $H:=G \mathrm{Wr} \operatorname{Sym}(2)$. This group acts primitively on the set $\Omega:=V \Gamma \times V \Gamma$, with all 
subdegrees finite. Choose vertices $\alpha$ and $\beta$ adjacent in $\Gamma$ and let $\Lambda$ be the orbital digraph $\left(\Omega,((\alpha, \alpha),(\alpha, \beta))^{H}\right)$. We shall denote the number of $H_{(\alpha, \alpha)}$-orbits in the sphere $S_{r}((\alpha, \alpha), \Lambda)$ by $n_{r}$, and the number of $H_{(\alpha, \alpha)}$-orbits in the ball $B_{r}((\alpha, \alpha), \Lambda)$ by $N_{r}$.

Since $G$ acts distance-transitively on $\Gamma, n_{r}=\lceil r / 2\rceil$, where $\lceil r / 2\rceil$ denotes the smallest integer greater than or equal to $r / 2$. Furthermore, the subdegrees of $H$ in the sphere $S_{r}((\alpha, \alpha), \Lambda)$ are $2 m(m-1)^{r-1} t^{r}$ and $2 m^{2}(m-1)^{r-2} t^{r}$ for $r \geq 2$, and, if $r$ is even, $m^{2}(m-1)^{r-2} t^{r}$.

The largest suborbit in the sphere $S_{r}((\alpha, \alpha), \Lambda)$ has size $2 m^{2}(m-1)^{r-2} t^{r}$, and for all sufficiently large integers $r$,

$$
2 m^{2}(m-1)^{r-2} t^{r} \leq(m-1)^{2 r-2} t^{2 r} .
$$

Thus, there exists an integer $R$ such that, for all $r>R$, the largest suborbit in $S_{r}((\alpha, \alpha), \Lambda)$ has cardinality strictly less than the cardinality of every suborbit in $\Lambda \backslash B_{2 r}((\alpha, \alpha), \Lambda)$.

Now consider the lower subdegree sequence $\left(m_{r}\right)$ of $H$ which, in this case, is equal to the subdegree sequence of $H$. Choose $r>R$ and find the largest integer $s \geq 2$ such that

$$
m_{s}=2 m^{2}(m-1)^{r-2} t^{r} .
$$

Our aim is to find the number of subdegrees that are less than or equal to $m_{s}$, and from this determine $s$.

Since $m_{s}$ is the largest suborbit in $B_{r}((\alpha, \alpha), \Lambda)$ we have $s \geq N_{r}$. Furthermore, $m_{s}$ is strictly less than the cardinality of every $(\alpha, \alpha)$-suborbit in $\Lambda \backslash B_{2 r}((\alpha, \alpha), \Lambda)$, so there can be at most $N_{2 r}$ suborbits with cardinality less than or equal to $m_{s}$. Hence $s \leq N_{2 r}$, and therefore $N_{r} \leq s \leq N_{2 r}$. Since $N_{r}=\sum_{i=1}^{r} n_{i}$, we have $N_{r} \geq r(r+1) / 4$ and $N_{r} \leq r(r+1) / 2$. Thus

$$
r(r+1) / 4 \leq s \leq r(2 r+1) .
$$

If the lower subdegree sequence of $H$ exhibits polynomial growth of degree $d \geq 1$ then there exist positive real numbers $c_{1}, c_{2}$ such that for all $k \geq 1$,

$$
c_{1} k^{d} \leq m_{k} \leq c_{2} k^{d} .
$$

However, for all $r>R$ we may choose a maximal integer $s$ with $m_{s}=2 m^{2}(m-1)^{r-2} t^{r}$. For all sufficiently large $r$ we therefore have $s^{d} \leq r^{d}(2 r+1)^{d} \leq 2 m^{2 d}(m-1)^{d(r-2)} t^{d r}$, so the lower subdegree growth rate of $H$ is not polynomial.

Although the growth of the lower subdegree sequence of $H$ is faster than polynomial growth, it is not exponential. Indeed, for all $r>R$ there exists an integer $s_{r}$ with $m_{s_{r}}=2 m^{2}(m-$ $1)^{r-2} t^{r}$ and $s_{r} \geq r(r+1) / 4$. Hence, for all $a>1$,

$$
\lim _{r \rightarrow \infty} \frac{m_{s_{r}}}{a^{s_{r}}} \leq \frac{2 m^{2}(m-1)^{r-2} t^{r}}{a^{r(r+1) / 4}}=0 .
$$

The group $H$ is thus an example of a group exhibiting subexponential, non-polynomial growth. The existence of such a group demonstrates that the list of possible growth rates given previously is not exhaustive.

\subsection{The average subdegree sequence}

At this point it is perhaps relevant to draw the reader's attention to another seemingly natural method for enumerating subdegrees, that neatly avoids the problems caused by subdegrees which occur infinitely often. Let $G$ act primitively on an infinite set $\Omega$, and suppose every subdegree of $G$ is finite. Fix an orbital digraph $\Gamma$ of $G$, and a vertex $\alpha \in \Omega$. Let $b_{r}(\alpha, \Gamma)$ be the ball-size $\left|B_{r}(\alpha, \Gamma)\right|$, and let $N_{r}(\alpha, \Gamma)$ be the number of suborbits of $G_{\alpha}$ in $B_{r}(\alpha, \Gamma)$. The sequence $\left(b_{r} / N_{r}\right)$ is then the average subdegree sequence of $G$ with respect to the digraph $\Gamma$. The growth of this sequence can be used as a measure of the subdegree growth of $G$.

The usefulness of average subdegree growth as a measure of subdegree growth is limited, however, by its dependence on the orbital digraph chosen. While it is in fact possible to 
explicitly bound this dependence, a further, and more serious, limitation is an inherent lack of subtlety. As an illustration, suppose we are given integers $m, t \geq 2$. The groups $G:=$ Aut $\Gamma\left(m, K_{t+1}\right)$ and $H:=G \mathrm{Wr} \operatorname{Sym}(2)$ acting on $\Omega:=V \Gamma\left(m, K_{t+1}\right)$ and $\Omega \times \Omega$ respectively are manifestly dissimilar: all non-diagonal orbital digraphs of the former have infinitely many ends; all non-diagonal orbital digraphs of the latter have just one. Their many differences are encapsulated by their lower subdegree growth rates, with $G$ exhibiting exponential growth, and $H$ subexponential non-polynomial growth. However, if one were instead to compare the average subdegree growth rates the groups, no such distinction is possible.

Indeed, define $\Gamma:=\Gamma\left(m, K_{t+1}\right)$ and $\Lambda:=\left(\Omega^{2},((\alpha, \alpha),(\alpha, \beta))^{H}\right)$, where $\alpha$ and $\beta$ are adjacent in $\Gamma$. The average subdegree growth rate of $G$ with respect to $\Gamma$ is exponential, with

$$
\begin{aligned}
\lim _{r \rightarrow \infty}\left(\frac{\left|B_{r}(\alpha, \Gamma)\right|}{N_{r}(\alpha, \Gamma)}\right)^{1 / r} & \geq\left(\frac{m(m-1)^{r-1} t^{r}}{r}\right)^{1 / r} \\
& =(m-1) t .
\end{aligned}
$$

Furthermore, $\left|B_{r}(\alpha, \Gamma)\right| \leq r\left|S_{r}(\alpha, \Gamma)\right|$, so the limit is equal to $(m-1) t$.

By Lemma 2.6,

$$
\left|S_{r}((\alpha, \alpha), \Lambda)\right|=\sum_{k=0}^{r}\left|S_{k}(\alpha, \Gamma)\right|\left|S_{r-k}(\alpha, \Gamma)\right|,
$$

so

$$
\left|B_{r}((\alpha, \alpha), \Lambda)\right| \leq \frac{m(r+1)\left|B_{r}(\alpha, \Gamma)\right|}{(m-1)} .
$$

Hence $\left|B_{r}(\alpha, \Gamma)\right| \leq\left|B_{r}((\alpha, \alpha), \Lambda)\right| \leq K(r+1)\left|B_{r}(\alpha, \Gamma)\right|$, where $K:=m /(m-1)$.

Two vertices $\left(\gamma_{1}, \gamma_{2}\right),\left(\delta_{1}, \delta_{2}\right) \in V \Lambda$ lie in the same $H_{(\alpha, \alpha)}$ orbit if and only if the sets $\left\{d_{\Gamma}\left(\alpha, \gamma_{1}\right), d_{\Gamma}\left(\alpha, \gamma_{2}\right)\right\}$ and $\left\{d_{\Gamma}\left(\alpha, \delta_{1}\right), d_{\Gamma}\left(\alpha, \delta_{2}\right)\right\}$ are equal. Thus if $n_{r}$ denotes the number of $(\alpha, \alpha)$-suborbits in $S_{r}((\alpha, \alpha), \Lambda)$ then $1 \leq n_{r} \leq r$, so

$$
r \leq N_{r}((\alpha, \alpha), \Lambda) \leq r^{2} .
$$

Whence the average subdegree growth of $H$ is exponential, with

$$
\lim _{r \rightarrow \infty}\left(\frac{\left|B_{r}(\alpha, \Gamma)\right|}{N_{r}(\alpha, \Gamma)}\right)^{1 / r}=(m-1) t .
$$

In light of these problems we will henceforth focus only on the relationship between the lower and upper subdegree growth rates of a group, and its structure.

\section{Subdegree growth and group structure}

In this section we give bounds on the growth of the lower and upper subdegree sequences of infinite subdegree finite primitive groups, and show that, when the growth of the lower subdegree sequences is fast enough, the rate of growth uniquely determines the group. Following this, we show that in many cases the number of permutation-ends of a group is determined by its multiset of subdegrees.

THEOREM 4.1. ([10]) Let $\Gamma$ be an infinite connected vertex- and arc-transitive digraph with finite but unequal in-valency and out-valency. Then there is an epimorphism $\varphi$ from the vertex set of $\Gamma$ to the set of integers $\mathbb{Z}$ such that $(\alpha, \beta)$ is an arc of $\Gamma$ only if $\varphi(\beta)=\varphi(\alpha)+1$. 
Corollary 4.2. Suppose $G$ is a primitive group of permutations of an infinite set $\Omega$, and every suborbit of $G$ is finite. If $\alpha \in \Omega$ and $\Delta(\alpha)$ and $\Delta^{*}(\alpha)$ are paired $\alpha$-suborbits then $|\Delta(\alpha)|=\left|\Delta^{*}(\alpha)\right|$.

Let $G$ be a primitive group of permutations of an infinite set $\Omega$, with every subdegree of $G$ finite, and suppose $\Gamma$ is an orbital digraph of $G$. Fix $\alpha \in \Omega$. An upper bound can be found for the growth of the lower subdegree sequences by bounding the growth of $s_{r}:=\left|S_{r}(\alpha, \Gamma)\right|$, since $G_{\alpha}$ fixes $S_{r}(\alpha, \Gamma)$ setwise.

LEMMA 4.3. If $\Gamma$ is an infinite locally finite primitive digraph then, for all $r \geq 1$,

$$
s_{r} \leq s_{1}\left(s_{1}-1\right)^{r-1} .
$$

Proof. Since $\Gamma$ is vertex-primitive, it is connected and every vertex has valency $s_{1}$. Thus for all $r>1$, any vertex in the sphere $S_{r}(\alpha, \Gamma)$ is connected to at least one vertex in $S_{r-1}(\alpha, \Gamma)$, and is therefore adjacent to at most $s_{1}-1$ vertices in $S_{r+1}$. Whence $s_{r} \leq s_{r-1}\left(s_{1}-1\right)$.

A corresponding bound is easily obtained for the growth of the upper subdegree sequence.

LEMma 4.4. Let $G$ be a subdegree finite primitive group of permutations of an infinite set $\Omega$. If $\left(M_{r}\right)$ is the upper subdegree sequence of $G$, then for all $r \geq 1$,

$$
M_{r} \leq 2 M_{1}\left(2 M_{1}-1\right)^{r-1} \text {. }
$$

Proof. Fix $\alpha \in \Omega$ and choose $\beta \in \Omega$ such that the suborbit $\beta^{G_{\alpha}}$ is of size $M_{1}$. Let $\Gamma$ be the orbital digraph $\left(\Omega,(\alpha, \beta)^{G}\right)$, and let $\Delta(\alpha)$ denote the suborbit $\beta^{G_{\alpha}}$.

If $\Delta^{*}(\alpha)$ is the suborbit paired with $\Delta(\alpha)$ then $\left|\Delta^{*}(\alpha)\right|$ is also equal to $M_{1}$ by Corollary 4.2 . Since $S_{1}=\Delta(\alpha) \cup \Delta^{*}(\alpha)$, the valency of $\Gamma$ is at most $2 M_{1}$.

If the upper subdegree sequence contains just one subdegree then there is nothing to prove, so suppose this is not the case. Choose $r \geq 1$ such that $M_{r}$ and $M_{r+1}$ are elements of the upper subdegree sequence of $G$; since all members of this sequence are distinct, there exists an integer $t \geq 1$ such that $M_{r}$ is the largest subdegree in the sphere $S_{t}$ but not in $S_{t+1}$. Thus, there exists a vertex $\gamma \in S_{t}$ such that $\gamma$ is adjacent to a vertex $\delta \in S_{t+1}$ with $\left|\delta^{G_{\alpha}}\right|>M_{r}$.

Since $\left|\delta^{G_{\alpha}}\right|>M_{r}$, we have $\left|\delta^{G_{\alpha}}\right| \geq M_{r+1}$, and therefore

$$
M_{r+1} \leq\left|\delta^{G_{\alpha}}\right| \leq\left(s_{1}-1\right)\left|\gamma^{G_{\alpha}}\right| \leq M_{r}\left(s_{1}-1\right) .
$$

If $\Gamma$ is a locally finite primitive digraph with connectivity one, and $T$ is the block-cut-vertextree of $\Gamma$, we define the lobe-distance between two vertices $\alpha$ and $\beta$ in $\Gamma$ to be

$$
b d(\alpha, \beta):=\frac{d_{T}(\alpha, \beta)}{2} .
$$

Informally, one may think of the lobe-distance between $\alpha$ and $\beta$ as being the number of lobes of $\Gamma$ through which any geodesic between $\alpha$ and $\beta$ must pass.

If $\Gamma$ is an infinite locally finite primitive digraph and $\alpha \in V \Gamma$, we define $n_{r}(\Gamma)$ to be the number of $\alpha$-suborbits of Aut $\Gamma$ in $S_{r}(\alpha, \Gamma)$. Since $G$ acts transitively on $\Gamma$, this definition is independent of our choice of $\alpha$. When there can be no ambiguity as to the identity of the digraph in question, $n_{r}(\Gamma)$ will be written as $n_{r}$. 
Let $\left\{f_{r}\right\}_{r \geq 0}$ be the sequence of Fibonacci numbers defined by the relation $f_{r+1}=f_{r}+f_{r-1}$ for $r \geq 1$ and $f_{0}=f_{1}=1$.

THEOREM 4.5. If $\Gamma$ is a locally finite primitive digraph with connectivity one that is not distance-transitive then $n_{r} \geq f_{r}$ for all $r \geq 1$.

Proof. Fix $\alpha \in V \Gamma$. To each vertex $\beta$ in $V \Gamma$ choose a geodesic in $\Gamma$ from $\alpha$ to $\beta$ and assign a label $\ell(\beta):=\left(l_{1}, \ldots, l_{k}\right)$, where $k=b d(\alpha, \beta)$, and for all $r$ satisfying $1 \leq r \leq k$ the integer $l_{r}$ is the number of vertices at lobe-distance $r$ from $\alpha$ in the geodesic. It is simple to check that, since $\Gamma$ has connectivity one, this is independent of the geodesic chosen. Let $L_{r}$ be the set of labels for vertices in $S_{r}(\alpha, \Gamma)$ and put $k_{r}:=\left|L_{r}\right|$. It is very easy to see two vertices $\beta, \beta^{\prime} \in S_{r}(\alpha, \Gamma)$ lie in the same $\alpha$-suborbit only if $\ell(\beta)=\ell\left(\beta^{\prime}\right)$. Thus $n_{r}(\Gamma) \geq k_{r}$.

By Theorem 1.2, if $\Gamma$ is not distance-transitive then the lobes of $\Gamma$ have diameter at least 2 . We claim that, in this case,

$$
k_{r+1} \geq k_{r}+k_{r-1} .
$$

Indeed, for all $r \geq 1$ there is an injective correspondence from $L_{r}$ into $L_{r+1}$ via the map sending $\left(l_{1}, \ldots, l_{k}\right) \in L_{r}$ to $\left(l_{1}, \ldots, l_{k}, 1\right) \in L_{r+1}$. Fix $r \geq 1$ and note there are $k_{r-1}$ labels in $L_{r}$ whose last entry is 1 . If $\left(l_{1}, \ldots, l_{k-1}, 1\right)$ is such a label, then $\left(l_{1}, \ldots, l_{k-1}, 2\right)$ is a label in $L_{r+1}$. Therefore, there are at least $k_{r-1}$ labels in $L_{r+1}$ whose final entry is 2 ; we have already seen there are $k_{r}$ labels in $L_{r+1}$ whose final entry is 1 , so we must have $k_{r+1} \geq k_{r}+k_{r-1}$, as claimed. Since $k_{0}=k_{1}=1$ we have $n_{r} \geq k_{r} \geq f_{r}$ for all $r \geq 0$.

It is well known that

$$
\lim _{r \rightarrow \infty} f_{r}^{1 / r}=\frac{1+\sqrt{5}}{2}
$$

consequently it is possible to find a lower bound for the growth of the sequence $\left(n_{r}^{1 / r}\right)$.

COROLlary 4.6. If $\Gamma$ is a locally finite primitive digraph with connectivity one that is not distance-transitive then

$$
\liminf _{r \rightarrow \infty} n_{r}^{1 / r} \geq \frac{1+\sqrt{5}}{2}
$$

The bound given in the above corollary is sharp; that is, it cannot be improved upon. Indeed, consider the Peterson graph $P_{5}$. This is a finite primitive distance-transitive graph with diameter 2 . It can be considered to be directed by inserting arcs, one in each direction, between adjacent vertices. Let $\Gamma:=\Gamma\left(2, P_{5}\right)$ and $G:=$ Aut $\Gamma$, and fix $\alpha \in V \Gamma$. It is hopefully clear that two vertices $\beta, \beta^{\prime} \in S_{r}(\alpha, \Gamma)$ lie in the same orbit of $G_{\alpha}$ if and only if $\ell(\beta)=\ell\left(\beta^{\prime}\right)$. Thus $n_{r}=k_{r}$. We claim, for all $r \geq 1$,

$$
k_{r+1}=k_{r}+k_{r-1} .
$$

Indeed, let $L_{r}^{\prime}$ be the elements of $L_{r}$ whose last entry is 1 , let $L_{r}^{\prime \prime}$ be those elements whose last entry is 2 and put $k_{r}^{\prime}:=\left|L_{r}^{\prime}\right|$ and $k_{r}^{\prime \prime}:=\left|L_{r}^{\prime \prime}\right|$. Since $P_{5}$ has diameter 2 we have $k_{r}=k_{r}^{\prime}+k_{r}^{\prime \prime}$. We may assign to each label $\ell \in L_{r}^{\prime \prime}$ the unique label in $L_{r-1}^{\prime}$ obtained by changing the last entry in $\ell$ from 2 to 1 . Hence $k_{r}^{\prime \prime} \geq k_{r-1}^{\prime}$. We may also assign to each label $\ell \in L_{r-1}^{\prime}$ a unique label in $L_{r}^{\prime \prime}$ obtained by changing the last entry in $\ell$ to 2 , so $k_{r-1}^{\prime} \geq k_{r}^{\prime \prime}$. Whence $k_{r}^{\prime \prime}=k_{r-1}^{\prime}$. Similarly, we assign to each label $\left(l_{1}, \ldots, l_{k}\right) \in L_{r-1}$ the label $\left(l_{1}, \ldots, l_{k}, 1\right) \in L_{r}^{\prime}$, and to each label $\left(l_{1}, \ldots, l_{k}, 1\right) \in L_{r}^{\prime}$ the label $\left(l_{1}, \ldots, l_{k}\right) \in L_{r-1}$. Such a correspondence is bijective, so $k_{r-1}=k_{r}^{\prime}$. Thus $k_{r+1}=k_{r+1}^{\prime}+k_{r+1}^{\prime \prime}=k_{r+1}^{\prime}+k_{r}^{\prime}=k_{r}+k_{r-1}$, as claimed. 
Now $k_{0}=k_{1}=1$ and so by (4.2), for all $r \geq 0$ we have $n_{r}=k_{r}=f_{r}$. Thus

$$
\lim _{r \rightarrow \infty} n_{r}^{1 / r}=\frac{1+\sqrt{5}}{2} .
$$

Let $\Gamma$ be a infinite locally finite connected vertex- and arc-transitive digraph, and fix $\alpha \in V \Gamma$. For $\gamma \in S_{r}(\alpha)$ we define

$$
\begin{aligned}
& a(\gamma):=\left|S_{1}(\alpha) \cap S_{r}(\gamma)\right| ; \\
& b(\gamma):=\left|S_{1}(\alpha) \cap S_{r+1}(\gamma)\right| \\
& c(\gamma):=\left|S_{1}(\alpha) \cap S_{r-1}(\gamma)\right| .
\end{aligned}
$$

The following lemma is an extension of an observation by Macpherson in [8]. The argument presented here is based on that given by Dicks and Dunwoody in $[\mathbf{3}]$.

Lemma 4.7. If there exists a natural number $R_{0}$ such that, for all $\beta, \gamma \in V \Gamma$ with $d(\alpha, \beta)>$ $R_{0}$ and $d(\alpha, \gamma)>R_{0}$, we have $c(\beta)=c(\gamma)$ and $b(\beta)=b(\gamma)$, then $\Gamma$ has more than one end.

Proof. Suppose, for all $\beta, \gamma \in V \Gamma$ with $d(\alpha, \beta)>R_{0}$ and $d(\alpha, \gamma)>R_{0}$, we have $c(\beta)=c(\gamma)$ and $b(\beta)=b(\gamma)$. We will describe an infinite set of vertices $s$, such that the complement $s^{*}:=$ $V \Gamma \backslash s$ is infinite, and the set of $\operatorname{arcs} \delta s$ from $s$ to $s^{*}$ is finite, thus showing $\Gamma$ must have more than one end.

Fix an arc $\left(\alpha_{0}, \alpha_{1}\right) \in V \Gamma$ and define $s:=\left\{\gamma \in V \Gamma \mid R_{0}+1 \leq d\left(\alpha_{0}, \gamma\right)=d\left(\alpha_{1}, \gamma\right)+1\right\}$. This set and its compliment are both infinite. Indeed, given a positive integer $n$ one may choose a vertex $\gamma \in V \Gamma$ with $d\left(\alpha_{0}, \gamma\right)=2 n+1$. There exists a geodesic $\alpha_{0} \beta_{1} \beta_{2} \ldots \beta_{2 n} \gamma$ of length $2 n+1$ between $\alpha_{0}$ and $\gamma$. If $e$ is the arc between $\beta_{n}$ and $\beta_{n+1}$ then, since $\Gamma$ is arc-transitive, there exists an automorphism of $\Gamma$ mapping $e$ to the arc $\left(\alpha_{0}, \alpha_{1}\right)$. Therefore, both $s$ and $s^{*}$ contain vertices in $S_{n}\left(\alpha_{0}, \Gamma\right) \cup S_{n+1}\left(\alpha_{0}, \Gamma\right)$. Whence $s$ and $s^{*}$ are infinite.

We now show $\delta s$ is finite. Suppose $e \in E$ is an arc between $\beta \in s$ and $\gamma \in s^{*}$. Write $i=$ $d\left(\alpha_{0}, \beta\right)$.

We claim $d\left(\alpha_{0}, \gamma\right)=1+d\left(\alpha_{1}, \gamma\right)$. Observe $d\left(\alpha_{0}, \gamma\right) \leq d\left(\alpha_{0}, \beta\right)+d(\beta, \gamma)=i+1$, and $d\left(\alpha_{0}, \beta\right) \leq d\left(\alpha_{0}, \gamma\right)+d(\beta, \gamma)$, so $d\left(\alpha_{0}, \gamma\right) \geq d\left(\alpha_{0}, \beta\right)-d(\beta, \gamma)=i-1$. Hence,

$$
i-1 \leq d\left(\alpha_{0}, \gamma\right) \leq i+1 \text {. }
$$

We now consider three cases: when $d\left(\alpha_{0}, \gamma\right)=i-1$, when $d\left(\alpha_{1}, \gamma\right)=i$ and finally when $d\left(\alpha_{0}, \gamma\right) \geq i$ and $d\left(\alpha_{1}, \gamma\right) \leq i-1$, and in each case show the claim is true.

Suppose $d\left(\alpha_{0}, \gamma\right)=i-1$. Then $d\left(\alpha_{0}, \beta\right)=d\left(\alpha_{0}, \gamma\right)+d(\gamma, \beta)$, so there is a geodesic from $\alpha_{0}$ to $\beta$ that contains $\gamma$. Hence

$$
S_{1}\left(\alpha_{0}\right) \cap S_{i-2}(\gamma) \subseteq S_{1}\left(\alpha_{0}\right) \cap S_{i-1}(\beta) ;
$$

however, since $i-1 \geq R_{0}$ we have $\left|S_{1}\left(\alpha_{0}\right) \cap S_{i-2}(\gamma)\right|=c(\gamma)=c(\beta)=\left|S_{1}\left(\alpha_{0}\right) \cap S_{i-1}(\beta)\right|$, so the two sets must be equal. Now $\alpha_{1} \in S_{1}\left(\alpha_{0}\right) \cap S_{i-1}(\beta)$, so $\alpha_{1} \in S_{1}\left(\alpha_{0}\right) \cap S_{i-2}(\gamma)$; that is, $d\left(\alpha_{1}, \gamma\right)=i-2=d\left(\alpha_{0}, \gamma\right)-1$ as claimed.

Next, suppose $d\left(\alpha_{1}, \gamma\right)=i$. Then $d\left(\alpha_{1}, \gamma\right)=d\left(\alpha_{1}, \beta\right)+d(\gamma, \beta)$, so there is a geodesic from $\alpha_{1}$ to $\gamma$ that contains $\beta$. Hence

$$
S_{1}\left(\alpha_{1}\right) \cap S_{i+1}(\gamma) \subseteq S_{1}\left(\alpha_{1}\right) \cap S_{i}(\beta) ;
$$

however, since $i-1 \geq R_{0}$ we have $\left|S_{1}\left(\alpha_{1}\right) \cap S_{i+1}(\gamma)\right|=b(\gamma)=b(\beta)=\left|S_{1}\left(\alpha_{1}\right) \cap S_{i}(\beta)\right|$, so the two sets must be equal. Now $\alpha_{0} \in S_{1}\left(\alpha_{1}\right) \cap S_{i}(\beta)$, so $\alpha_{0} \in S_{1}\left(\alpha_{1}\right) \cap S_{i+1}(\gamma)$; that is, $d\left(\alpha_{0}, \gamma\right)=$ $i+1=d\left(\alpha_{1}, \gamma\right)+1$ as claimed. 
Finally, if $d\left(\alpha_{0}, \gamma\right) \geq i$ and $d\left(\alpha_{1}, \gamma\right) \leq i-1$, then $d\left(\alpha_{1}, \gamma\right)+1 \leq i \leq d\left(\alpha_{0}, \gamma\right)$. In fact, since $d\left(\alpha_{0}, \gamma\right) \leq d\left(\alpha_{1}, \gamma\right)+d\left(\alpha_{1}, \alpha_{0}\right)=d\left(\alpha_{1}, \gamma\right)+1$, we have $d\left(\alpha_{0}, \gamma\right) \geq 1+d\left(\alpha_{1}, \gamma\right)$, so $d\left(\alpha_{0}, \gamma\right)=$ $1+d\left(\alpha_{1}, \gamma\right)$ as claimed.

Now $\gamma \in s^{*}$, so either $d\left(\alpha_{0}, \gamma\right)<R_{0}+1$ or $d\left(\alpha_{0}, \gamma\right) \neq d\left(\alpha_{1}, \beta\right)+1$. The latter is not true by the above argument, so we must have $d\left(\alpha_{0}, \gamma\right)<R_{0}+1$. Hence, there are only finitely many $\gamma \in s^{*}$ that are adjacent to a vertex in $s$, so $\delta s$ is finite, and $\Gamma$ has more than one end.

THEOREM 4.8. If $\Gamma$ is an infinite vertex- and arc-transitive locally finite digraph with one end then $n_{r}(\Gamma) \geq 2$ for all large enough $r$.

Proof. Let $\Gamma$ be an infinite locally finite digraph with one end, and fix $\alpha \in V \Gamma$. We claim there is an integer $R_{0}$ such that for all $r \geq R_{0}$ there exist vertices $\gamma_{1}, \gamma_{2} \in S_{r}(\alpha)$ with $c\left(\gamma_{1}\right) \neq$ $c\left(\gamma_{2}\right)$ or $b\left(\gamma_{1}\right) \neq b\left(\gamma_{2}\right)$.

Suppose no such $R_{0}$ exists. Then there exists an infinite sequence $\left(r_{i}\right)$ such that for all $\gamma_{1}, \gamma_{2} \in S_{r_{i}}(\alpha)$ we have $c\left(\gamma_{1}\right)=c\left(\gamma_{2}\right)$ and $b\left(\gamma_{1}\right)=b\left(\gamma_{2}\right)$. For each $i \geq 1$ choose $\gamma \in S_{r_{i}}(\alpha)$ and set $c_{r_{i}}:=c(\gamma)$ and $b_{r_{i}}:=b(\gamma)$. It is easy to see $c_{r_{i}} \geq c_{r_{i-1}}$ and $b_{r_{i}} \leq b_{r_{i-1}}$. Since $1 \leq c_{r_{i}} \leq$ $\left|S_{1}(\alpha)\right|$ and $1 \leq b_{r_{i}} \leq\left|S_{1}(\alpha)\right|$ for all $i \geq 0$, there exists constants $k, c$ and $b$ such that, for all $i \geq k$ we have $c_{r_{i}}=c$ and $b_{r_{i}}=b$. Put $R_{0}:=r_{k}$. Suppose there exists $r \geq R_{0}$ such that $S_{r}(\alpha)$ contains two vertices $\gamma_{1}$ and $\gamma_{2}$ with $c\left(\gamma_{1}\right) \neq c\left(\gamma_{2}\right)$. Without loss of generality, one may suppose $c\left(\gamma_{1}\right)>c\left(\gamma_{2}\right)$. However, $r>R_{0}$ so $c\left(\gamma_{2}\right) \geq c$ and $c\left(\gamma_{1}\right)>c$. If $i$ is chosen so $r_{i}>r$ then $c_{r_{i}} \geq c\left(\gamma_{1}\right)>c$ which is a contradiction.

It must therefore be the case that, for all $r \geq R_{0}$, we have $c(\gamma)=c$ for all $\gamma \in S_{r}(\alpha)$. A similar argument shows that for all $r \geq R_{0}$, we have $b(\gamma)=b$ for all $\gamma \in S_{r}(\alpha)$. Hence, by Lemma 4.7, the digraph $\Gamma$ must have more than one end. Since this is not the case, our claim must be true.

Let $G:=$ Aut $\Gamma$. It is clear that two vertices $\gamma_{1}, \gamma_{2} \in S_{r}(\alpha)$ lie in the same orbit of $G_{\alpha}$ only if $c\left(\gamma_{1}\right)=c\left(\gamma_{2}\right)$ and $b\left(\gamma_{1}\right)=b\left(\gamma_{2}\right)$. Hence, for all $r>R_{0}$ we have $n_{r}(\Gamma) \geq 2$.

THEOREM 4.9. Let $G$ be a primitive group of permutations of an infinite set $\Omega$. If $G$ is subdegree finite with more than one permutation-end then the lower subdegree sequence of $G$ grows exponentially if and only if $G$ is distance-transitive.

In this case, $G$ acts distance-transitively on some distance-transitive infinite locally finite digraph $\Gamma\left(m, K_{t+1}\right)$, and the subdegree sequence $\left(m_{r}\right)$ of $G$ has height $\omega$ and satisfies

$$
\lim _{r \rightarrow \infty} m_{r}^{1 / r}=\left(\frac{m-1}{m}\right) m_{1} .
$$

Furthermore, if the growth of the lower subdegree sequence of $G$ is not exponential, then it is polynomial.

Proof. Suppose $G$ is a primitive group of permutations of an infinite set $\Omega$, possessing an orbital digraph with more than one end, and $G$ has a finite suborbit whose pair is also finite. By Theorem 1.1, every suborbit of $G$ is finite. Let $\left(m_{r}\right)$ be the lower subdegree sequence of $G$. Note that, if $G$ acts distance-transitively on the locally finite infinite distance-transitive digraph $\Gamma\left(m, K_{t_{1}+1}\right)$, then every suborbit is self-paired, $G$ has height $\omega$, so $\left(m_{r}\right)$ is equal to the subdegree sequence of $G$, and the subdegree growth of $G$ is exponential with

$$
\begin{aligned}
\lim _{r \rightarrow \infty} m_{r}^{1 / r} & =\left(\frac{m-1}{m}\right) m t_{1} \\
& =\left(\frac{m-1}{m}\right) m_{1} .
\end{aligned}
$$


Now consider the converse. Suppose the group $G$ does not act distance-transitively on any locally finite infinite distance-transitive digraph. We will show that the lower subdegree growth of $G$ is bounded above by some polynomial.

By Corollary 4.6, $\liminf n_{r}^{1 / r} \geq(1+\sqrt{5}) / 2>3 / 2$. Hence, there exists an integer $R$ such that, for all $r>R$, we have $n_{r}>(3 / 2)^{r}$, and thus $N_{r}>(3 / 2)^{r}$.

Fix $r>R$ and observe that $m_{N_{r}} \leq s_{1}\left(s_{1}-1\right)^{r-1}$ by Lemma 4.3. We may choose an integer $N$ such that, for all $n \geq N$, we have $(3 / 2)^{n} \geq s_{1}\left(s_{1}-1\right)$. Thus, for all $n \geq N$,

$$
m_{N_{r}}<N_{r}^{n} \text {. }
$$

Furthermore, given any integer $s$ with $N_{r-1} \leq s \leq N_{r}$, the subdegree $m_{s}$ satisfies $m_{s} \leq$ $s_{1}\left(s_{1}-1\right)^{r-1}$. Since $s \geq N_{r-1}$ we also have $s^{n} \geq N_{r-1}^{n}>(3 / 2)^{n(r-1)}>s_{1}\left(s_{1}-1\right)^{r-1} \geq m_{s}$, so the growth of the lower subdegree sequence of $G$ is polynomial.

If one removes the condition that $G$ have more than one permutation-end then the following is obtained.

THEOREM 4.10. Suppose $G$ is an infinite subdegree finite group of permutations of an infinite set $\Omega$, and $G$ is not distance-transitive. If $\left(m_{r}\right)$ is the lower subdegree sequence of $G$ then

$$
\liminf _{r \rightarrow \infty} m_{r}^{1 / r} \leq \sqrt{2 m_{1}-1}
$$

Proof. Suppose $G$ is a subdegree finite primitive group of permutations of an infinite set $\Omega$ and does not act distance-transitively on any digraph. If $G$ has an orbital digraph with more than one end then its lower subdegree growth is subexponential by Theorem 4.9. So, suppose $G$ has an orbital digraph with precisely one end; every orbital digraph of $G$ therefore has one permutation-end. Let $\Delta(\alpha)$ be a suborbit of size $m_{1}$, and let $\Gamma$ be the orbital digraph $(\Omega, \Delta)$. Then $S_{1}(\alpha, \Gamma)=\Delta(\alpha) \cup \Delta^{*}(\alpha)$. Let $s_{r}:=\left|S_{r}(\alpha, \Gamma)\right|$ and $n_{r}:=n_{r}(\Gamma)$, and let $N_{r}$ be the sum $\sum_{i=1}^{r} n_{i}$. Since all suborbits of $G$ are finite, one may deduce from Corollary 4.2 that $\Delta^{*}(\alpha)$, the suborbit paired with $\Delta(\alpha)$, also has cardinality $m_{1}$. Thus, $s_{1} \leq 2 m_{1}$.

From the proof of Theorem 4.9, $m_{N_{r}} \leq s_{1}\left(s_{1}-1\right)^{r-1}$. We again note it is sufficient to show this result holds when $G=$ Aut $\Gamma$.

By Theorem 4.8, there exists an integer $R_{0}$ such that $n_{r} \geq 2$ for all $r \geq R_{0}$. Hence, for sufficiently large $r$,

$$
N_{r} \geq R_{0}+2\left(r-R_{0}\right) .
$$

Thus, if $a>1$,

$$
\begin{aligned}
\limsup _{r \rightarrow \infty} m_{N_{r}}^{1 / N_{r}} & \leq \limsup _{r \rightarrow \infty}\left(s_{1}-1\right)^{r / N_{r}} \\
& \leq \limsup _{r \rightarrow \infty}\left(s_{1}-1\right)^{r /\left(2 r-R_{0}\right)} \\
& =\sqrt{2 m_{1}-1} .
\end{aligned}
$$

Hence $\liminf _{r \rightarrow \infty} m_{r}^{1 / r} \leq \sqrt{2 m_{1}-1}$ as required.

CoROllary 4.11. If $G$ is a group acting primitively on an infinite set $\Omega$ with a finite suborbit whose pair is also finite, and $\left(m_{r}\right)$ is the lower subdegree sequence of $G$, then

$$
\liminf _{r \rightarrow \infty} m_{r}^{1 / r}>\sqrt{2 m_{1}-1}
$$


if and only if $G$ acts distance-transitively on some distance-transitive locally finite infinite digraph $\Gamma\left(m, K_{t+1}\right)$ with $m>2$ and $t \geq 2$, or $m=2$ and $t \geq 4$.

Proof. Suppose $G$ is a subdegree finite primitive group of permutations of an infinite set $\Omega$. If $G$ does not act distance-transitively on any locally finite orbital digraph and $\left(m_{r}\right)$ is the lower subdegree sequence of $G$ then

$$
\liminf _{r \rightarrow \infty} m_{r}^{1 / r} \leq \sqrt{2 m_{1}-1}
$$

by Theorem 4.10 .

Now suppose $G$ acts distance-transitively on a locally finite distance-transitive digraph $\Gamma$. By Theorem 1.2 , we may write $\Gamma=\Gamma\left(m, K_{t+1}\right)$ for some $m \geq 2$ and $t \geq 2$. Observe that $\liminf _{r \rightarrow \infty} m_{r}^{1 / r}=(m-1) t$, so the limit $\liminf _{r \rightarrow \infty} m_{r}^{1 / r}>\sqrt{2 m_{1}-1}$ precisely when $m>2$ and $t \geq 2$, or $m=2$ and $t \geq 4$.

All known examples of primitive groups with locally finite one-ended orbital digraphs exhibit subexponential lower subdegree growth; furthermore, it seems highly unlikely that examples exhibiting exponential growth exist.

CONJECTURE 4.12. If $G$ is a group acting primitively on an infinite set $\Omega$ with a finite suborbit whose pair is also finite, then $G$ has exponential lower subdegree growth if and only if $G$ is distance-transitive.

Of course there are many further questions that remain unanswered. Which primitive groups exhibit subexponential non-polynomial subdegree growth, and are there gaps in growth rates that allow one to determine a group given its subdegree growth rate? What is the relationship between the permutation-ends of a group and its subdegree growth rates? This latter question is the focus of the final section of this paper. In it, we detail some preliminary results that illustrate a relationship does indeed exist; however, much work remains to be done before its nature is fully determined.

\section{Subdegree growth and ends of orbital digraphs}

We begin by observing that the relationship between the subdegree growth of a primitive group $G$ and its permutation-end structure is more subtle than one might expect.

THEOREM 5.1. If $\left(m_{r}\right)$ is the lower subdegree sequence of a subdegree finite infinite primitive group $G \leq \operatorname{Sym}(\Omega)$, then there exist infinite primitive groups $G^{\prime}$ and $G^{\prime \prime}$ whose suborbits are all finite, with lower subdegree sequences $\left(m_{r}^{\prime}\right)$ and $\left(m_{r}^{\prime \prime}\right)$ respectively, such that $G^{\prime}$ has one permutation-end, and $G^{\prime \prime}$ has infinitely many permutation-ends, with

$$
m_{r}^{\prime} \leq 2 m_{r}
$$

and

$$
m_{r}^{\prime \prime} \leq 2 m_{r}
$$

for all $r \geq 1$.

Proof. Fix $\alpha \in \Omega$, and write $\underline{\alpha}:=(\alpha, \alpha) \in \Omega^{2}$. Take $G^{\prime}$ to be the wreath product $G \mathrm{Wr}$ Sym (2), and consider its product action on $\Omega^{2}$. By Theorem 2.4, all suborbits of $G^{\prime}$ 
are finite and every orbital digraph has one end. Furthermore, for every suborbit $\beta^{G_{\alpha}}$ of $G$, the set $(\beta, \alpha)^{G_{\underline{\alpha}}^{\prime}}$ is a suborbit of $G^{\prime}$; since $\left|(\beta, \alpha)^{G_{\underline{\alpha}}^{\prime}}\right|=2\left|\beta^{G_{\alpha}}\right|$, we have $m_{r}^{\prime} \leq 2 m_{r}$.

Let $G^{\prime \prime}$ be the group $G(2, G)$ constructed in Chapter 2.2, and let $\Lambda$ be an orbital digraph of $G$ acting on $\Omega$ with connectivity greater than one; by Theorem $2.3, G^{\prime \prime}$ acts primitively on the vertex set of the digraph $\Gamma(2, \Lambda)$. Indeed, this digraph is an orbital digraph of $G^{\prime \prime}$. Since $\Gamma(2, \Lambda)$ has infinitely many ends, every orbital digraph of $G^{\prime \prime}$ has infinitely many permutation-ends. Furthermore, the action of $G_{\{\Lambda\}}^{\prime \prime}$ on $V \Lambda$ is isomorphic to the action of $G$ on $V \Lambda=\Omega$; thus, for each subdegree $m_{r}$ of $G$, the group $G_{\alpha,\{\Lambda\}}^{\prime \prime}$ has an orbit on $V \Lambda$ of size $m_{r}$. Hence, for each subdegree $m_{r}$ of $G$, the group $G^{\prime \prime}$ has a suborbit of size $2 m_{r}$. Whence, $m_{r}^{\prime \prime} \leq 2 m_{r}$.

It should be noted that both the difference between $m_{r}$ and $m_{r}^{\prime}$, and the difference between $m_{r}$ and $m_{r}^{\prime \prime}$, may grow arbitrarily large, leaving gaps in the range of possible rates of growth.

Certain growth rates are only exhibited by groups with precisely one permutation-end. The following is immediate from Theorem 4.9.

THEOREM 5.2. If $G$ is a subdegree finite primitive group of permutations of an infinite set $\Omega$, and the lower subdegree growth of $G$ is subexponential but not polynomial, then $G$ has precisely one permutation-end.

Example 7 shows primitive groups with non-polynomial but subexponential lower subdegree growth exist. All known examples of subdegree finite primitive groups with exponential lower subdegree growth have infinitely many permutation-ends. Indeed, it seems highly likely that such growth rates cannot be achieved by groups with just one permutation-end. If true then the above theorem and the following conjecture would allow one to naturally partition the nonpolynomial rates of growth of infinite primitive groups according to the number of permutationends possessed by each group.

CONJECTURE 5.3. If $G$ is an infinite primitive permutation group whose subdegrees are all finite, and the lower subdegree sequence of $G$ grows exponentially, then $G$ has $2^{\aleph_{0}}$ permutationends.

In Section 3.1 it was shown that there exist examples of infinite primitive groups with precisely one permutation-end, and infinite primitive groups with infinitely many permutationends, both possessing bounded lower subdegree sequences. If, instead of examining just the lower subdegree sequence, one considers the whole subdegree sequence, it is again possible to determine the permutation-end structure of those infinite primitive groups exhibiting specific rates of subdegree growth.

We begin with two theorems describing the structure of primitive groups with more than one permutation-end.

ThEOREM 5.4. [11, Theorem 2.5] Let $G$ be a vertex-transitive group of automorphisms of a connectivity-one digraph $\Gamma$ whose lobes have at least three vertices, and let $T$ be the blockcut-vertex tree of $\Gamma$. If there exist distinct vertices $\alpha, \beta \in V \Gamma$ such that, for some vertex $x \in$ $(\alpha, \beta)_{T}$,

$$
G_{\alpha, x}=G_{\beta, x},
$$

then $G$ does not act primitively on $V \Gamma$. 
THEOREM 5.5. [12, Theorem 3.11] If $G$ is a primitive group of permutations of an infinite set $\Omega$ with more than one permutation-end and no infinite subdegree, then $G$ has a locally finite orbital digraph $\Gamma$ with connectivity one, whose lobes are primitive but not automorphismregular, are pairwise isomorphic, have at least three vertices and at most one end. Furthermore, if $\Lambda$ is a lobe of $\Gamma$, then $G_{\{\Lambda\}}$ acts primitively but not regularly on $V \Lambda$.

Using these results, it is possible to determine precisely the permutation-end structure of primitive groups whose subdegrees are bounded above.

THEOREM 5.6. If $G$ is an infinite primitive permutation group whose subdegrees are all finite and bounded above, then $G$ has precisely one permutation-end.

Proof. Suppose $G$ is an infinite primitive permutation group with more than one permutation-end whose subdegrees are all finite. By Theorem 5.5, $G$ has an orbital digraph of the form $\Gamma(m, \Lambda)$ for some integer $m \geq 2$, and for some primitive digraph $\Lambda$. Let $\Gamma$ denote the digraph $\Gamma(m, \Lambda)$ and let $T$ be the block-cut-vertex tree of $\Gamma$.

Observe that if $\alpha$ and $\beta$ are vertices in $\Gamma$ and $x \in V T$ lies on the $T$-geodesic $[\alpha, \beta]_{T}$ between $\alpha$ and $\beta$, then $G_{\alpha, \beta} \leq G_{x}$ and therefore

$$
\left|G_{\alpha}: G_{\alpha, \beta}\right|=\left|G_{\alpha}: G_{\alpha, x}\right|\left|G_{\alpha, x}: G_{\alpha, \beta}\right| .
$$

Hence, the cardinality of the suborbit $\beta^{G_{\alpha}}$ is equal to the product $\left|x^{G_{\alpha}}\right|\left|\beta^{G_{\alpha, x}}\right|$.

Thus, if all subdegrees of $G$ are bounded above, then there exists a finite number $k$ such that any automorphism in $G$ fixing the sphere $S_{k}(\alpha, \Gamma)$ pointwise must also fix every vertex in $\Gamma$, and therefore every vertex in $T$. However, groups with this property cannot be primitive by Theorem 5.4.

Using a similar argument, it is sometimes possible to determine the permutation-end structure of a primitive group by knowing just one subdegree.

THEOREM 5.7. If $G$ is an infinite primitive permutation group whose subdegrees are all finite, and at least one subdegree is prime, then every orbital digraph of $G$ has precisely one end.

Proof. Again suppose that $G$ is an infinite primitive permutation group with more than one permutation-end whose subdegrees are all finite. Let $\Gamma$ be a connectivity-one orbital digraph of $G$ of the form $\Gamma(m, \Lambda)$, the existence of which is assured by Theorem 5.5, and let $T$ be the block-cut-vertex tree of this digraph.

Recall that, given $\alpha, \beta \in V \Gamma$ and a vertex $x \in V T$ lying on the $T$-geodesic $[\alpha, \beta]_{T}$ between $\alpha$ and $\beta$, the cardinality of the suborbit $x^{G_{\alpha}}$ is equal to $\left|x^{G_{\alpha}}\right|\left|\beta^{G_{\alpha, x}}\right|$.

Since $G$ acts arc-transitively on $\Gamma$, it permutes the lobes of $\Gamma$. By Theorem 5.5, the setwise stabiliser in $G$ of each lobe acts primitively on the vertices of the lobe; whence, for each vertex $\alpha \in V \Gamma$, the stabiliser $G_{\alpha}$ transitively permutes the lobes of $\Gamma$ that contain $\alpha$. Thus $G_{\alpha}$ acts transitively on the sphere $S_{1}(\alpha, T)$, which has cardinality $m \geq 2$.

If $\Lambda$ is a lobe of $\Gamma$, then by Theorem 5.5, $\Lambda$ has at least three vertices, and $G_{\{\Lambda\}}$ acts primitively but not regularly on $V \Lambda$. Thus if $\alpha \in V \Lambda$, then $G_{\alpha,\{\Lambda\}}$ fixes no vertex in $\Lambda \backslash \alpha$. The lobe $\Lambda$ corresponds to some vertex $x \in S_{1}(\alpha, T)$, so $G_{\alpha, x}$ fixes no vertex in $S_{1}(x, T) \backslash\{\alpha\}$. Therefore, for each vertex $\gamma$ in $S_{1}(x, T) \backslash\{\alpha\}$, there exists a prime number $p$ dividing the cardinality of the orbit $\gamma^{G_{\alpha, x}}$. Hence $m p$ divides $\gamma^{G_{\alpha}}$. 
Since $G_{\alpha}$ acts transitively on the sphere $S_{1}(\alpha, T)$, for all vertices $\gamma$ in $S_{2}(\alpha, T)$ there exist primes $p$ and $q$ such that $p q$ divides the subdegree $\left|\gamma^{G_{\alpha}}\right|$; therefore, the same is true of all vertices in $T$ lying at distance greater than two from $\alpha$. Since this includes all vertices in $\Gamma \backslash\{\alpha\}$, no subdegree of $G$ is prime.

This brief look at the relationship between the subdegree growth rates and the permutationend structure of infinite subdegree finite primitive groups contained an obvious omission: the class of groups that exhibit polynomial subdegree growth. Groups with just one permutationend, and groups with infinitely many permutation-ends, are well represented in this class. It would be extremely interesting to know if it is possible to determine the permutation-end structure of such groups from the order of their growth.

It appears that more must be known about the structure of locally finite graphs with precisely one end before any significant progress in this area can be made. A clear insight into their nature would also aid the construction of a proof of, or counterexample to, Conjecture 5.3.

Many of the results in this paper are taken from the author's DPhil thesis, completed at the University of Oxford, under the supervision of Peter Neumann. The author would like to thank Dr Neumann for his enthusiasm and insightful suggestions. The author would also like to thank the EPSRC for their generous funding.

\section{References}

1. S. A. Adeleke and P. M. Neumann, 'Relations related to betweenness: their structure and automorphisms', Mem. Amer. Math. Soc. (623) 131 (1998).

2. P. J. Cameron, Oligomorphic permutation groups, London Mathematical Society Lecture Notes Series 152 (Cambridge University Press, Cambridge, 1990).

3. W. Dicks and M. J. Dunwoody, Groups acting on graphs, Cambridge Studies in Advanced Mathematics 17 (Cambridge University Press, Cambridge, 1989).

4. J. Dixon and B. Mortimer, Permutation groups, Graduate Texts in Mathematics 163 (Springer-Verlag, New York, 1996).

5. D. M. Evans, 'Suborbits in infinite primitive permutation groups', Bull. London Math. Soc. (5) 33 (2001) 583-590.

6. W. Imrich and N. Seifter, 'A note on the growth of transitive graphs', Discrete Math. (1-2) 73 (1988/89) 111-117.

7. A. A. Ivanov, 'Bounding the diameter of a distance-regular graph', Dokl. Akad. Nauk SSSR (4) 28 (1983) 789-792.

8. H. D. Macpherson, 'Infinite distance transitive graphs of finite valency', Combinatorica (1) 2 (1982) 63-69.

9. A. Yu. Ol'Shanskiǔ, Geometry of defining relations in groups, Mathematics and its Applications (Soviet Series) 70 (Kluwer Acad. Publ., Dordrecht, 1991).

10. C. E. Praeger, 'On homomorphic images of edge-transitive directed graphs', Australas. J. Combin. 3 (1991) 207-210.

11. S. M. Smith, 'Infinite primitive directed graphs', J. Algebraic Combin. (31) 1 (2010) 131-141.

12. S. M. Smith, 'Orbital graphs of infinite primitive permutation groups', J. Group Theory (10) 6 (2007) $817-828$.

13. V. I. Trofimov, 'Graphs with polynomial growth', Math. USSR Sbornik (2) 51 (1985) 405-417.

14. M. E. Watkins, 'Ends and automorphisms of infinite graphs', Graph symmetry (eds G. Hahn and G. Sabidussi), NATO Adv. Sci. Inst. Ser. C Math. Phys. Sci. 497 (Kluwer Acad. Publ., Dordrecht, 1997), pp. 379-414.

Simon M. Smith

Mathematical Institute

University of Oxford

Oxford

UK
Current address:

Department of Mathematics

Syracuse University

Syracuse, New York

$U S A$

simon.smith@chch.oxon.org 